\title{
Optimal control in a malaria model: intervention of fumigation and bed nets
}

\author{
Bevina D. Handari', Febyan Vitra', Radhiya Ahya', Tengku Nadya S. ${ }^{1}$ and Dipo Aldila1*
}

${ }^{*}$ Correspondence:

aldiladipo@sci.ui.ac.id

'Department of Mathematics, Universitas Indonesia, Depok, Indonesia

\section{Springer}

\begin{abstract}
Malaria is one of the world's most serious health problems because of the increasing number of cases every year. First, we discuss a deterministic model of epidemic SIR-SI spread of malaria with the intervention of bed nets and fumigation. We found that the malaria-free equilibrium is locally asymptotically stable (LAS) when $\mathcal{R}_{0}<1$ and unstable otherwise. A malaria endemic equilibrium exists and is LAS when $\mathcal{R}_{0}>1$. Sensitivity analysis of $\mathcal{R}_{0}$ shows that the use of bed nets and fumigation can reduce $\mathcal{R}_{0}$. We modify the previous model into a stochastic differential equation model to understand the effect of random environmental factors on the spread of malaria. Numerical simulations show that when $\mathcal{R}_{0}>1$, a greater value of noise intensity $\sigma$ generates a solution that is different from a deterministic solution; when $\mathcal{R}_{0}<1$, regardless of the $\sigma$ value, the solution approaches a deterministic solution. Then the deterministic model was modified into an optimal control model to determine the best strategy in controlling the spread of malaria by using fumigation as the control variable. Numerical simulations show that periodic fumigations cost less than constant intervention and can reduce the number of infected humans. Priority is given to the endemic prevention strategy rather than to the endemic reduction strategy. For more effective intervention, the value of $\mathcal{R}_{0}$ should receive close attention. A potentially endemic $\left(\mathcal{R}_{0}>1\right)$ environment requires more frequent fumigation than an environment that is not potentially endemic $\left(\mathcal{R}_{0}<1\right)$. A combination of the use of bed nets and fumigation can reduce the number of infected individuals at minimal cost.
\end{abstract}

Keywords: Malaria; Optimal control problem; Fumigation; Stochastic differential equation

\section{Introduction}

Malaria is a dangerous infectious disease caused by a Plasmodium parasite, which can be transmitted to humans through bites from infected Anopheles female mosquitos. The symptoms usually appear after one to two weeks and include fever, sweating, shivering or cold, vomiting, headache, diarrhea, and muscle aches (Infodatin [14]). Based on the 2017 World Malaria Report, the number of malaria cases in the world increased to 216 million in 2016. Those cases were mostly located in Africa (90\%), Southeast Asia (7\%), and the Eastern Mediterranean (2\%) (WHO [22]). From 2015 to 2016, malaria cases in Indonesia reached 217,025 and were mostly found in Papua, Papua Barat, West Nusa Tenggara, Maluku, and North Maluku (Infodatin [14]).

(c) The Author(s) 2019. This article is distributed under the terms of the Creative Commons Attribution 4.0 International License (http://creativecommons.org/licenses/by/4.0/), which permits unrestricted use, distribution, and reproduction in any medium, provided you give appropriate credit to the original author(s) and the source, provide a link to the Creative Commons license, and indicate if changes were made. 
There are many methods of preventing malaria, the most popular of which are using bed nets at night to prevent mosquito bites and using fumigation to reduce local mosquito populations (WHO [22]). In recent studies, researchers have constructed mathematical models to analyze malaria spread. These include mathematical models of malaria transmission that consider climatic factors (Abebe et al. [4]), mathematical models of malaria distribution by using mosquito nets (Agusto et al. [1]; Chitnis et al. [8]; Ngonghala et al. [18]), and mathematical models of climate-based malaria with the use of mosquito nets (Xiunan and Xiao-Qiang [25]), to which this paper refers.

Several factors should be considered when attempting to eliminate malaria vectors, such as climate factors. In tropical areas, there are differences between mosquito life expectancy in the dry and rainy seasons. Dembele et al. [9] state that there is a greater percentage of deaths from malaria during the rainy season than during the dry season. Therefore this climate factor will be considered in the model.

In this paper, we first include two malaria preventatives to the model, the use of bed nets without insecticides and fumigation. First, we construct a deterministic model of the spread of malaria with both fumigation and bed nets. We then determine the equilibrium point and basic reproduction number $\mathcal{R}_{0}$ followed by numerical simulations to analyze how both means of intervention affect the human population.

In practice, several factors have been found to affect the spread of malaria, such as human factors (body temperature and carbon dioxide content released by the body) as described by Keyser [15] and residential factors (living close to stagnant water) as described by Theresa et al. [19]. Both factors are influenced by unpredictable environmental factors that cannot be explained by the deterministic model. Therefore the deterministic model is extended to a malaria model with stochasticity factors. Next, the stochastic model is discussed by Gray et al. [12], and numerical simulations are implemented to evaluate the dynamics of stochastic factors in spreading malaria throughout the population.

However, some obstacles arose due to the use of fumigation, such as high costs and adverse effects of continuous fumigation on the environment. Hence we ultimately developed the deterministic model into an optimal control problem. Then we analyzed the best strategy for controlling the spread of malaria by using fumigation at minimal cost.

\section{Malaria deterministic model with fumigation and bed nets}

There are two mathematical models for the spread of malaria in humans: the susceptible infected recovered (SIR) model is used on the human population $(h)$, and the susceptible infected (SI) model is used on the Anopheles mosquito population ( $v$ ). The difference between the two models lies in the recovered $(R)$ compartment, which only humans possess; Anopheles mosquitos' lifespans are too short for them to enter this stage. Both human and mosquito populations were assumed to be homogeneous closed populations; thus the total populations of humans $N_{h}$ and mosquitos $N_{v}$ can be considered to be the sums of all compartments of each population. Malaria may cause deaths in the human population. According to WHO [23], in 2017, there were estimated 435,000 deaths from malaria globally, compared with 451,000 estimated deaths in 2016. The WHO African region accounted for $93 \%$ of all malaria deaths in 2017 , but it also accounted for $88 \%$ of the 172,000 fewer global malaria deaths reported in 2017 compared with 2010. All WHO regions except the WHO region of the America's recorded reductions in mortality in 2017 compared with 2010. Our proposed model in this paper aims to understand the behavior of malaria 
only in a short time period with considering a short time period of intervention. Therefore we put aside the death rate due to malaria from our model. With this assumption, we have that all human deaths are considered natural. Fumigation and the use of bed nets were used in the models as means of intervention to eliminate malaria with the assumption that no mosquitos are resistant to fumigation. Note that the long-term intervention of fumigation may lead to genetic mutation of mosquitoes as described by Bustamam, Aldila, and Yuwanda [6].

The malaria infection process is assumed to happen with successful infection probabilities $c_{h}$ and $c_{v}$ and with mosquito bite rates of $\beta_{h}$ and $\beta_{v}$. The use of bed nets can decrease the total number of mosquito bites. However, since not all people use bed nets at home, the mosquito bite rate is defined as follows:

$$
\beta_{i}(k, \eta)=\beta_{i} k \eta+\beta_{i}(1-k)
$$

where $k$ indicates the proportion of humans who use bed net, and $\eta$ indicates the effectiveness of the bed nets. An increase in $k$ indicates the increasing number of people using bed nets. On the other hand, an increase in $\eta$ results in poorer prevention of mosquito bites related to the quality of the bed nets. Note that $\eta$ and $k$ are bounded parameters in $[0,1]$. Human and mosquito deaths in the model are only caused by natural deaths $\mu_{h}$ and $\mu_{v}$, respectively, although mosquito deaths are also caused by fumigation $u$.

The recovery rate of humans is $\gamma$ and can revert to being susceptible at rate $\delta$. The flow diagram model is seen in Fig. 1.

According to Fig. 1, the SIR-SI mathematical model of malaria disease spread with fumigation and bed nets is as follows:

$$
\begin{aligned}
& \frac{d S_{h}}{d t}=A_{h}-\frac{c_{h} \beta_{h}(k, \eta) S_{h} I_{v}}{N_{h}}-\mu_{h} S_{h}+\delta R_{h}, \\
& \frac{d I_{h}}{d t}=\frac{c_{h} \beta_{h}(k, \eta) S_{h} I_{v}}{N_{h}}-\gamma I_{h}-\mu_{h} I_{h},
\end{aligned}
$$

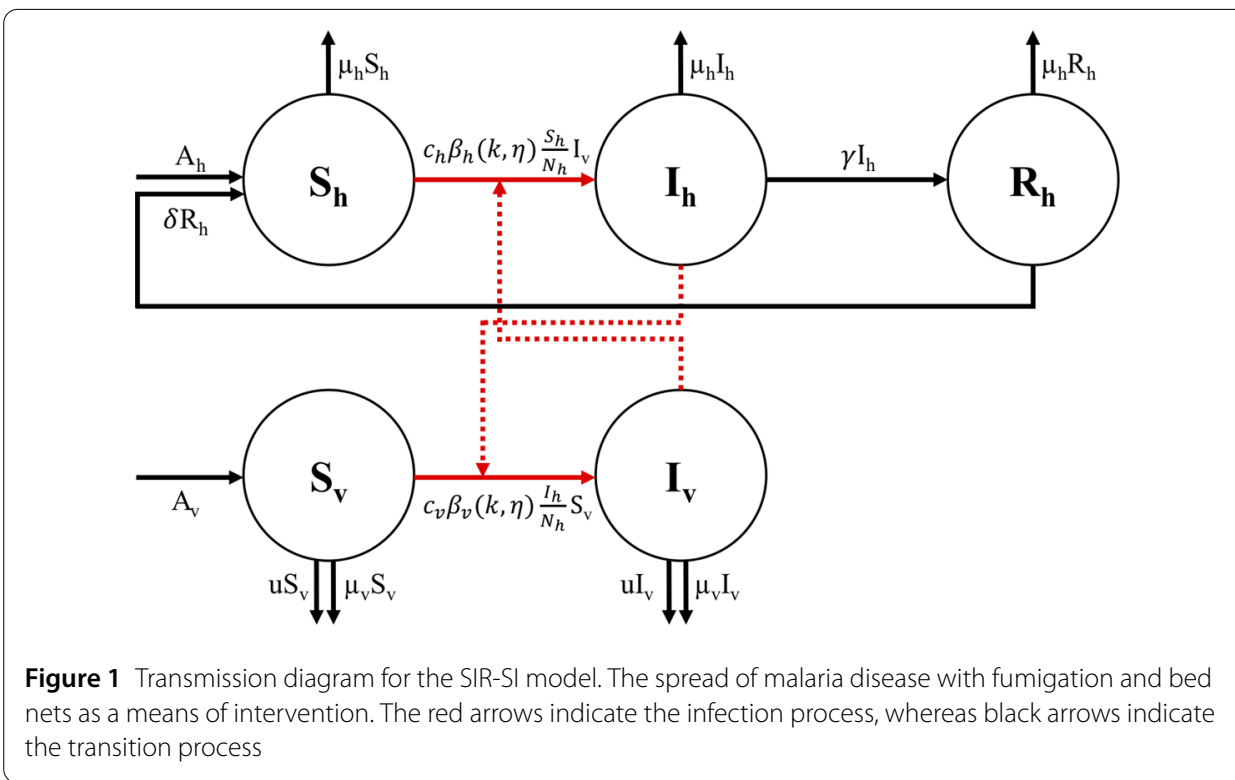


Table 1 Parameters of SIR-SI model (1)

\begin{tabular}{|c|c|c|c|c|}
\hline No & Par & Description & Condition & Dimension \\
\hline 1 & $A_{h}$ & Daily human birth rate & $A_{h}>0$ & person $\times$ day $^{-1}$ \\
\hline 2 & $A_{V}$ & Daily mosquito birth rate & $A_{V}>0$ & mosquito $\times$ day $^{-1}$ \\
\hline 3 & $\mu_{h}$ & Natural death rate of humans & $\mu_{h}>0$ & $\operatorname{day}^{-1}$ \\
\hline 4 & $\mu_{v}$ & Natural death rate of mosquitos & $\mu_{v}>0$ & day $^{-1}$ \\
\hline 5 & $\gamma$ & Recovery rate of humans & $\gamma>0$ & day $^{-1}$ \\
\hline 6 & $\delta$ & $\begin{array}{l}\text { Rate of transition back to vulnerable humans due } \\
\text { to the end of the immunity period }\end{array}$ & $\delta>0$ & day $^{-1}$ \\
\hline 7 & $c_{h}$ & $\begin{array}{l}\text { Successful infection probability from infectious } \\
\text { mosquitos to susceptible humans }\end{array}$ & $c_{h}>0$ & $(\text { mosquito } \times \text { day })^{-1}$ \\
\hline 8 & $c_{V}$ & $\begin{array}{l}\text { Successful infection probability from susceptible } \\
\text { mosquitos to infectious humans }\end{array}$ & $c_{V}>0$ & $(\text { mosquito } \times \text { day })^{-1}$ \\
\hline 9 & $\beta_{h}$ & Biting rate & $\beta_{h} \geq 1$ & $\frac{\text { person }}{\text { mosquitoxday }}$ \\
\hline 10 & $\beta_{\vee}$ & Biting rate & $\beta_{V} \geq 1$ & day $^{-1}$ \\
\hline 11 & $u$ & Fumigation rate & $u \geq 0$ & day $^{-1}$ \\
\hline 12 & k & Bed net proportion & $0 \leq k \leq 1$ & - \\
\hline 13 & $\eta$ & Bed net quality & $0<\eta<1$ & - \\
\hline
\end{tabular}

$$
\begin{aligned}
& \frac{d R_{h}}{d t}=\gamma I_{h}-\delta R_{h}-\mu_{h} R_{h}, \\
& \frac{d S_{v}}{d t}=A_{v}-\frac{c_{v} \beta_{v}(k, \eta) S_{v} I_{h}}{N_{h}}-\mu_{v}(t) S_{v}-u(t) S_{v}, \\
& \frac{d I_{v}}{d t}=\frac{c_{v} \beta_{v}(k, \eta) S_{v} I_{h}}{N_{h}}-\mu_{v}(t) I_{v}-u(t) I_{v}
\end{aligned}
$$

where $\mu_{v}(t)$ and $u(t)$ are functions depending on time, which describe seasonality and fumigation in mosquito population, respectively. Later we will discuss further details about the dependency of these parameter on time. For the analysis of model (1) related to their equilibrium point and their basic reproduction number, we assume that $u(t)=u$ and $\mu_{v}(t)=\mu_{v}$ as constant parameters. The description of parameters in model (1) is given in Table 1. Since we assume that without fumigation intervention, the total human and mosquito populations are constant, we have that

$$
\frac{d\left(S_{h}+I_{h}+R_{h}\right)}{d t}=\frac{d N_{h}}{d t}=A_{h}-\mu_{h} N_{h}, \quad 0=A_{h}-\mu_{h} N_{h},
$$

which gives us $N_{h}=\frac{A_{h}}{\mu_{h}}$, that is, $A_{h}=\mu_{h} N_{h}$. On the other hand, for mosquito population, we have

$$
\frac{d\left(S_{v}+I_{v}\right)}{d t}=\frac{d N_{v}}{d t}=A_{v}-\mu_{v} N_{v}, \quad 0=A_{v}-\mu_{v} N_{v}
$$

which gives us $N_{v}=\frac{A_{v}}{\mu_{v}}$, that is, $A_{v}=\mu_{v} N_{v}$. When $u \neq 0$, we have that

$$
\frac{d\left(S_{v}+I_{v}\right)}{d t}=\frac{d N_{v}}{d t}=A_{v}-\mu_{v} N_{v}-u N_{v}=-u N_{v}<0,
$$

which indicates that the total number of mosquitoes decreases with respect to time.

Next, we analyze the equilibrium points and the corresponding basic reproduction number $\mathcal{R}_{0}$. The equilibrium points of the model are the malaria-free equilibrium (MFE) and malaria endemic equilibrium (MEE). We end the analytic discussion with a discussion of the stability of equilibrium points. 
MFE represents a condition where no individual is infected with malaria. Furthermore, MFE is also used to analyze $\mathcal{R}_{0}$. From model (1) we obtain MFE as follows:

$$
\begin{aligned}
\mathrm{MFE} & =\left(S_{h}^{*}, I_{h}^{*}, R_{h}^{*}, S_{v}^{*}, I_{v}^{*}\right) \\
& =\left(\frac{A_{h}}{\mu_{h}}, 0,0, \frac{A_{v}}{u+\mu_{v}}, 0\right) .
\end{aligned}
$$

MFE has positive values on $\mathbb{R}_{5}^{+}$, which means that it always exists biologically.

(a) The basic reproduction number $\mathcal{R}_{0}$ is used to analyze whether the malaria is endemic $\left(\mathcal{R}_{0} \geq 1\right)$ or not $\left(\mathcal{R}_{0}<1\right)$. $\mathcal{R}_{0}$ can be constructed using the next-generation matrix (NGM) method [10]. Please see [2, 3, 6] for further examples of the construction of the NGM for epidemic models. First, we construct the Jacobian matrix of infected compartments constructed from system (1):

$$
J=\left[\begin{array}{cc}
-\mu_{h}-\gamma & \frac{c_{h} \beta_{h}(k, \eta) S_{h}}{N_{h}} \\
\frac{c_{v} \beta_{v}(k, \eta) S_{v}}{N_{h}} & -u-\mu_{v}
\end{array}\right] .
$$

The matrix $J$ is decomposed into a transmission matrix $T$ that contains the infectious parameter and a transition matrix $V$ that does not contain the infectious parameter as follows:

$$
\begin{aligned}
& T=\left[\begin{array}{cc}
0 & \frac{c_{h} \beta_{h}(k, \eta) S_{h}}{N_{h}} \\
\frac{c_{v} \beta_{v}(k, \eta) S_{v}}{N_{h}} & 0
\end{array}\right], \\
& V=\left[\begin{array}{cc}
-\left(\gamma+\mu_{h}\right)^{-1} & 0 \\
0 & -\left(u+\mu_{v}\right)^{-1}
\end{array}\right] .
\end{aligned}
$$

Therefore NGM is written as

$$
\mathrm{NGM}=-T V^{-1}=\left[\begin{array}{cc}
0 & \frac{c_{h} \beta_{h}(k, \eta) A_{h}}{\mu_{h} N_{h}\left(u+\mu_{\nu}\right)} \\
\frac{c_{\nu} \beta_{\nu}(k, \eta) A_{\nu}}{\left(\mu_{\nu}+u\right) N_{h}\left(\gamma+\mu_{h}\right)} & 0
\end{array}\right],
$$

and $\mathcal{R}_{0}$ is the spectral radius of NGM,

$$
\mathcal{R}_{0}=\sqrt{\frac{c_{h} \beta_{h}(k, \eta)}{\gamma+\mu_{h}} \frac{c_{v} \beta_{v}(k, \eta)}{u+\mu_{v}} \frac{N_{v}}{N_{h}}},
$$

where $N_{h}=\frac{A_{h}}{\mu_{h}}$ and $N_{v}=\frac{A_{v}}{u+\mu_{v}}$. Further discussion about $\mathcal{R}_{0}$ will be given later in this section.

(b) MEE represents a condition in which malaria always persists in a population. The MEE of the model is as follows:

$$
\begin{aligned}
\operatorname{MEE} & =\left(S_{h}^{* *}, I_{h}^{* *}, R_{h}^{* *}, S_{v}^{* *}, I_{v}^{* *}\right) \\
& =\left(\frac{A_{h}}{\mu_{h}}-\left(I_{h}^{* *}+R_{h}^{* *}\right), C_{1}\left(\mathcal{R}_{0}^{2}-1\right), C_{2}\left(\mathcal{R}_{0}^{2}-1\right), \frac{A_{v}}{\mu_{v}+u}-I_{v}^{* *}, C_{3}\left(\mathcal{R}_{0}^{2}-1\right)\right)
\end{aligned}
$$


with

$$
\begin{array}{ll}
C_{1}=\frac{\left(\delta+\mu_{h}\right) K}{c_{v} \beta_{v} \mu_{h}}, & C_{2}=\frac{\gamma K}{c_{v} \beta_{v} \mu_{h}}, \\
C_{3}=\frac{\left(\delta+\mu_{h}\right) K}{c_{h} \beta_{h}\left(u+\mu_{v}\right)}, & K=\frac{\left(\gamma+\mu_{h}\right)\left(u+\mu_{v}\right) N_{h}}{c_{h} \beta_{h} \gamma A_{v}+N_{h} \mu_{h}^{2}\left(u+\mu_{v}\right)} .
\end{array}
$$

According to Eq. (5), MEE exists when $\mathcal{R}_{0}>1$.

(c) The stability of the two equilibrium points can be determined with eigenvalue analysis from a system evaluated at the corresponding equilibrium point. To determine the stability of MFE, system (1) must be linearized on MFE as follows:

$$
J_{\mathrm{DFE}}=\left(\begin{array}{ccccc}
-\mu_{h} & 0 & \delta & 0 & -\frac{c_{h} \beta_{h} A_{h}}{\mu_{h} N_{h}} \\
0 & -\gamma-\mu_{h} & 0 & 0 & \frac{c_{h} \beta_{h} A_{h}}{\mu_{h} N_{h}} \\
0 & \gamma & -\delta-\mu_{h} & 0 & 0 \\
0 & -\frac{c_{v} \beta_{v} A_{v}}{\left(\mu_{v}+u\right) N_{h}} & 0 & -u-\mu_{v} & 0 \\
0 & \frac{c_{v} \beta_{v} A_{v}}{\left(\mu_{v}+u\right) N_{h}} & 0 & 0 & -u-\mu_{v}
\end{array}\right)
$$

where the characteristic polynomial of (6) is

$$
\left(\lambda+\delta+\mu_{h}\right)\left(\lambda+\mu_{h}\right)\left(\lambda+u+\mu_{v}\right)\left(a \lambda^{2}+b \lambda+c\right)=0
$$

with

$$
\begin{aligned}
& a=N_{h}^{2} \mu_{h}\left(u+\mu_{v}\right), \\
& b=N_{h}^{2} \mu_{h}\left(u+\mu_{v}\right)\left(\mu_{v}+\gamma+\mu_{h}+u\right), \\
& c=N_{h}^{2} \mu_{h}\left(\mu_{v}+u\right)^{2}\left(\gamma+\mu_{h}\right)\left(1-\mathcal{R}_{0}^{2}\right) .
\end{aligned}
$$

According to Verhulst [20], the MFE is asymptotically stable when $\operatorname{Re}\left(\lambda_{i}\right)<0$,

$\forall i=1,2, \ldots, n$. From Eq. (7) it follows that the MFE is asymptotically stable when $\mathcal{R}_{0}<1$.

Next, we analyze the stability of the MEE point. Substituting the MEE into the Jacobian matrix of system (1), we obtain five eigenvalues, with two of them $\lambda_{1}=-\left(\mu_{v}+u\right)$ and $\lambda_{2}=$ $-\mu_{h}$. The other three eigenvalues are taken from the root of the third-degree polynomial given by

$$
\sum_{i=0}^{3} a_{i} \lambda^{i}=0
$$

where

$$
\begin{aligned}
& a_{3}=1, \\
& a_{0}=\left(\delta+\mu_{h}\right)\left(\gamma+\mu_{h}\right)\left(u+\mu_{v}\right)\left(\mathcal{R}_{0}^{2}-1\right),
\end{aligned}
$$

whereas $a_{2}$ and $a_{1}$ have long expressions, which cannot be written explicitly in this paper. Nonetheless, we have that $a_{3}$ and $a_{2}$ are always positive, and $a_{0}>0$ when $\mathcal{R}_{0}>1$. According 
Table 2 Parameter values of SIR-SI model (1)

\begin{tabular}{rllll}
\hline No & Parameter & Value & Dimension & Reference \\
\hline 1 & $N_{h}$ & 10,000 & mosquito & Assumption \\
2 & $N_{V}$ & 10,000 & mosquito & Assumption \\
3 & $\mu_{h}$ & 0.000039 & day $^{-1}$ & Kim et al. [16] \\
4 & $\mu_{V}$ & 0.1 & day $^{-1}$ & CDC [7] \\
5 & $\gamma$ & 0.0035 & day $^{-1}$ & Chitnis et al. [8] \\
6 & $\delta$ & 0.00274 & day $^{-1}$ & Chitnis et al. [8] \\
7 & $C_{h}$ & 0.24 & mosquito $\times$ day $^{-1}$ & Chitnis et al. [8] \\
8 & $C_{V}$ & 0.024 & mosquito $\times$ day $^{-1}$ & Chitnis et al. [8] \\
9 & $\beta_{h}$ & 0.35 & person & CDC [7] \\
10 & $\beta_{V}$ & 0.35 & day $^{-1}$ & CDC [7] \\
11 & $u$ & To be evaluated & day $^{-1}$ & \\
12 & $k$ & To be evaluated & - & \\
13 & $\eta$ & To be evaluated & - & \\
\hline
\end{tabular}

to the Routh-Hurwitz stability criteria, all roots of (8) are negative if $a_{2}>0, a_{0}>0$, and $a_{2} a_{1}>a_{0}$. Therefore we have that the MEE of system (1) is locally asymptotically stable if $\mathcal{R}_{0}>1$ and $a_{2} a_{1}>a_{0}$. On the other hand, when $\mathcal{R}_{0}<1$ or $a_{2} a_{1} \leq a_{0}$, the MEE is unstable.

To understand the role of model parameters to the basic reproduction number $\mathcal{R}_{0}$, we analyze the sensitivity of $\mathcal{R}_{0}$ and perform autonomous simulation of the given intervention parameter. According to Kim et al. [16], both simulations use the initial conditions $S_{h}(0)=$ $0.5148 N_{h}, I_{h}(0)=0.2113 N_{h}, R_{h}(0)=N_{h}-S_{h}(0)-I_{h}(0), S_{v}(0)=N_{v}-I_{v}(0), I_{v}(0)=0.3267 N_{v}$, and the parameter values used are listed in Table 2.

Sensitivity analysis of $\mathcal{R}_{0}$ is implemented because it is related to policies that might be used by related parties. The first analysis is the $\mathcal{R}_{0}$ sensitivity of the mosquito biting rate toward humans, $\beta_{h}(k, \eta)$ with $k=0$, and without the use of bed nets. The second analysis is $\mathcal{R}_{0}$ sensitivity toward the fumigation rate $u$. An analytical study on $\mathcal{R}_{0}$ can be obtained as follows:

$$
\begin{aligned}
& \frac{\partial \mathcal{R}_{0}}{\partial \beta_{h}(0, \eta)}=\frac{1}{2} \sqrt{\frac{c_{h} c_{v} \beta_{v} N_{v}}{\beta_{h}\left(\gamma+\mu_{h}\right)\left(u+\mu_{v}\right) N_{h}}}>0, \\
& \frac{\partial \mathcal{R}_{0}}{\partial u}=-\frac{1}{2\left(u+\mu_{v}\right)} \sqrt{\frac{c_{h} c_{v} \beta_{v} N_{v}}{\left(\gamma+\mu_{h}\right)\left(u+\mu_{v}\right) N_{h}}}<0 .
\end{aligned}
$$

Equation (9a) is always positive, which means that the curve of the biting rate parameter toward $\mathcal{R}_{0}$ is increasing monotonically, or as $\beta_{h}$ increases, $\mathcal{R}_{0}$ also increases. On the other hand, Eq. (9b) is always negative, which means that the curve of the fumigation rate parameter toward $\mathcal{R}_{0}$ is decreasing monotonically, or as $u$ increases, $\mathcal{R}_{0}$ decreases.

When we substitute all parameters value from Table 2 into $\mathcal{R}_{0}=1$, we have $\frac{0.238673939 \sqrt{\beta_{h}}}{u+0.1}=1$. Figure 2 explains how $\mathcal{R}_{0}$ can be determined by relying on $u$ and $\beta_{h}$ qualitatively based on the previous equation. It can be seen from Fig. 2 that when $\beta_{h}<0.17554563$, the population always achieves a malaria-free situation $\left(\mathcal{R}_{0}<1\right)$, and thus fumigation is not yet needed. When $\beta_{h}>0.175545635$, fumigation is needed to reach $\mathcal{R}_{0}<1$. By solving $\frac{0.238673939 \sqrt{\beta_{h}}}{u+0.1}=1$ with respect to $u$ we have

$$
u_{\min }=0.238673929 \sqrt{\beta_{h}}-0.1
$$


Figure $2 \mathcal{R}_{0}$ sensitivity diagram is shown as a height curve of the parameters $\beta_{h}$ and $u$ with the critical line $\mathcal{R}_{0}=1$. The red colored area defines the endemic condition of malaria, whereas the blue colored area defines a condition with no malaria

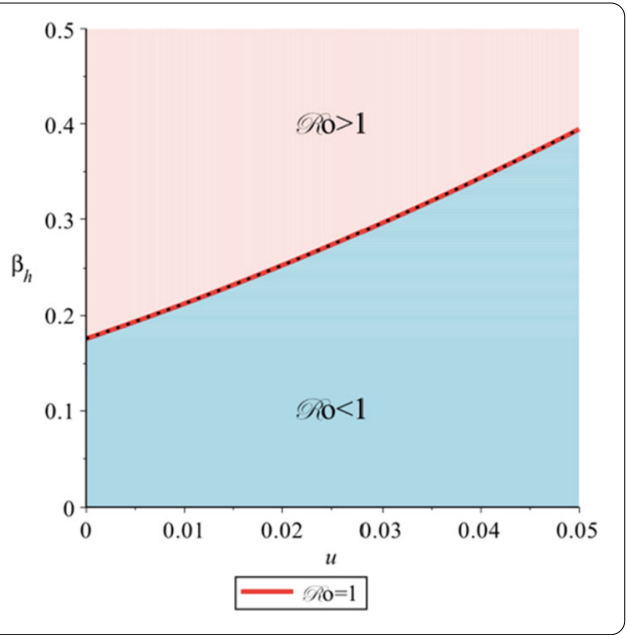

Therefore, when $\beta_{h}>0.175545635$, we need $u>u_{\min }$ to achieve the condition $\mathcal{R}_{0}<1$. This study indicates that there is a possibility that fumigation is not needed to eliminate malaria from humans. It is shown through the above simulation that when the infection rate is lower than the minimum boundary of $u$ that yields $\mathcal{R}_{0}<1$, fumigation is not needed. Furthermore, there are other parameters to consider when fumigation should be implemented in the field to yield $\mathcal{R}_{0}<1$, which is an infection rate.

The second analysis is $\mathcal{R}_{0}$ sensitivity toward the bed net parameter, which is the proportion of the users $k$ and the proportion of bed net effectiveness $\eta$. The analytical study on $\mathcal{R}_{0}$ is as follows:

$$
\begin{aligned}
& \frac{\partial \mathcal{R}_{0}}{\partial k}=-\sqrt{\frac{c_{h} \beta_{h} c_{v} \beta_{v}(1-\eta)^{2} N_{v}}{\left(\gamma+\mu_{v}\right)\left(u+\mu_{v}\right) N_{h}}}, \\
& \frac{\partial \mathcal{R}_{0}}{\partial \eta}=\sqrt{\frac{c_{h} \beta_{h} c_{v} \beta_{v} k N_{v}}{\left(\gamma+\mu_{v}\right)\left(u+\mu_{v}\right) N_{h}}} .
\end{aligned}
$$

According to Table 1 , the parameter $\eta$ is in the interval $(0,1)$, and $k$ is in the interval $[0,1]$; thus we can guarantee that $(\eta-1)<0$ and $(1-k) \geq 0$. This means that Eq. (11a) shows that $\mathcal{R}_{0}$ decreases monotonically with respect to $k$; that is, as $k$ increases, $\mathcal{R}_{0}$ decreases. Equation (11b) shows that $\mathcal{R}_{0}$ monotonically increases with respect to $\eta$; thus, as $\eta$ increases, $\mathcal{R}_{0}$ also increases.

Using the same approach as in Fig. 2, we substitute all parameters values from Table 2 except $k$ and $\eta$, which gives us $4.034325733 \sqrt{(0.35(k \eta-k+1))^{2}}=1$. Figure 3 explains how $\mathcal{R}_{0}$ can be determined by relying on $k$ and $\eta$ qualitatively. Figure 3 also further elucidates the threshold of $\mathcal{R}_{0}$; the red colored area defines a condition with $\mathcal{R}_{0}>1$, and the blue colored area defines a condition with $\mathcal{R}_{0}<1$.

It can be seen in Fig. 3 that when $k \leq k^{*}=0.2917917277$, the bed net usage intervention does not eliminate malaria; thus the proportion of bed net users must endeavor to achieve $k>k^{*}$. In other words, the government needs to distribute bed nets to more than $29.18 \%$ of the total endemic population. As the proportion of bed net user is less than $29.18 \%$, bed nets are not considered effective. Nonetheless, when using bed nets, the quality of the bed nets is an important consideration. According to Fig. 3, when $k>k^{*}$, the quality of 
Figure $3 \mathcal{R}_{0}$ sensitivity diagram is shown as the height curve from parameters $k$ and $\eta$ with the critical line $\mathcal{R}_{0}=1$. The red colored area defines the endemic condition of malaria, whereas the blue colored area defines a condition with no malaria

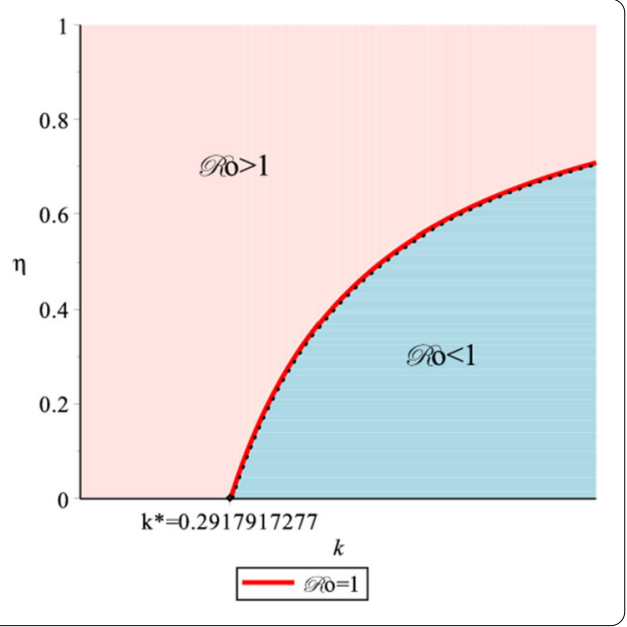

the bed nets is important to achieve $\mathcal{R}_{0}<1$. Solving $4.034325733 \sqrt{(0.35(k \eta-k+1))^{2}}=1$ with respect to $\eta$, we have

$$
\eta_{\min }=\frac{k-0.2917917277}{k}
$$

Therefore, when $k>k^{*}$, we need $\eta>\eta_{\min }$ to achieve the condition $\mathcal{R}_{0}<1$. This means that when providing bed nets, the quality of the bed nets needs to be considered. The better the quality of the bed nets, the higher the chance that the bed nets decrease the number of mosquito bites; thus the proportion of bed net use is lower when $k \simeq k^{*}$.

The life expectancy of mosquitos is different during the rainy season than in the dry one, making their natural death rates different as well. The natural death rate depends on time or $\mu_{v}(t)$. For example, the rainy season happens in the first six months followed by the dry season in the next six months on the interval $[0,365]$. Assuming that the natural death of mosquitos in the dry season is twice that in the rainy season, the natural death of mosquitos can be formulated as follows:

$$
\mu_{v}(t) \begin{cases}\mu_{1}=0.1, & 0<t<\frac{365}{2} \\ \mu_{2}=0.2, & \frac{365}{2}<t<365\end{cases}
$$

where $\mu_{1}$ is the natural death rate of the mosquito during the rainy season, and $\mu_{2}$ is the natural death rate during the dry season. In real circumstances, there is a transition season between both seasons. Therefore Eq. (13) needs to be changed into a continuous function by using a Fourier series (Wrede and Spiegel [24]). By including the transition season the natural death rate of mosquitos can be stated as follows:

$$
\mu_{v}(t)=0.15-0.06366197724 \sin \left(\frac{2}{365} \pi t\right)-0.02122065908 \sin \left(\frac{6}{365} \pi t\right) .
$$

Changes in the natural death rate of mosquitos shown in (14) also cause changes in the total population of the infected mosquitos. As a result, fumigation needs to be adjusted depending on the season. Because the life expectancy of mosquitos is higher during the rainy season, fumigation with substances that are more effective in killing mosquitos should be 
selected; in other words, the fumigation rate in the rainy season is higher than the rate during the dry season. Next, to compare the impacts of different types of fumigation, we performed an autonomous simulation for compartment models with differences in the fumigation parameter $u$. The simulation excludes the bed net intervention $(k=0)$. The first fumigation parameter has a constant fumigation rate $u=0.05$. The second fumigation rate is given periodically on the first day of the month by considering the season in Eq. (13) for 720 days, and the fumigation rates during the dry and rainy seasons are $u_{1}=1$ and $u_{2}=2$, respectively. The simulation does not include fumigation.

Fumigation is expected to suppress the mosquito populations so that there will be no vectors that can spread malaria. In Fig. 4, there is a quite a significant difference in the curves with fumigation compared to the curve without fumigation. Since no fumigation is implemented (blue curve), the number of susceptible humans decreased, which is caused by the high intensity of infections and is confirmed by the increased numbers of infected humans. On the other hand, the natural number of mosquitos decreases with or without fumigation. However, the implementation of fumigation accelerates the decrease of infected mosquitos and suppresses the endemic point. The plot on the bottom right of Fig. 4 shows the differences between no intervention, constant fumigation, and two seasons fumigation and shows a significant drop in the green curve due to a difference in the fumigation rates. With seasons, the total population of the mosquito tends to zero with $\mathcal{R}_{0}=0.9413426708<1$, and the epidemic eventually disappears. However, with no intervention, the value of $\mathcal{R}_{0}$ becomes $\mathcal{R}_{0}=1.412014006>1$; in other words, the endemic state still exists.

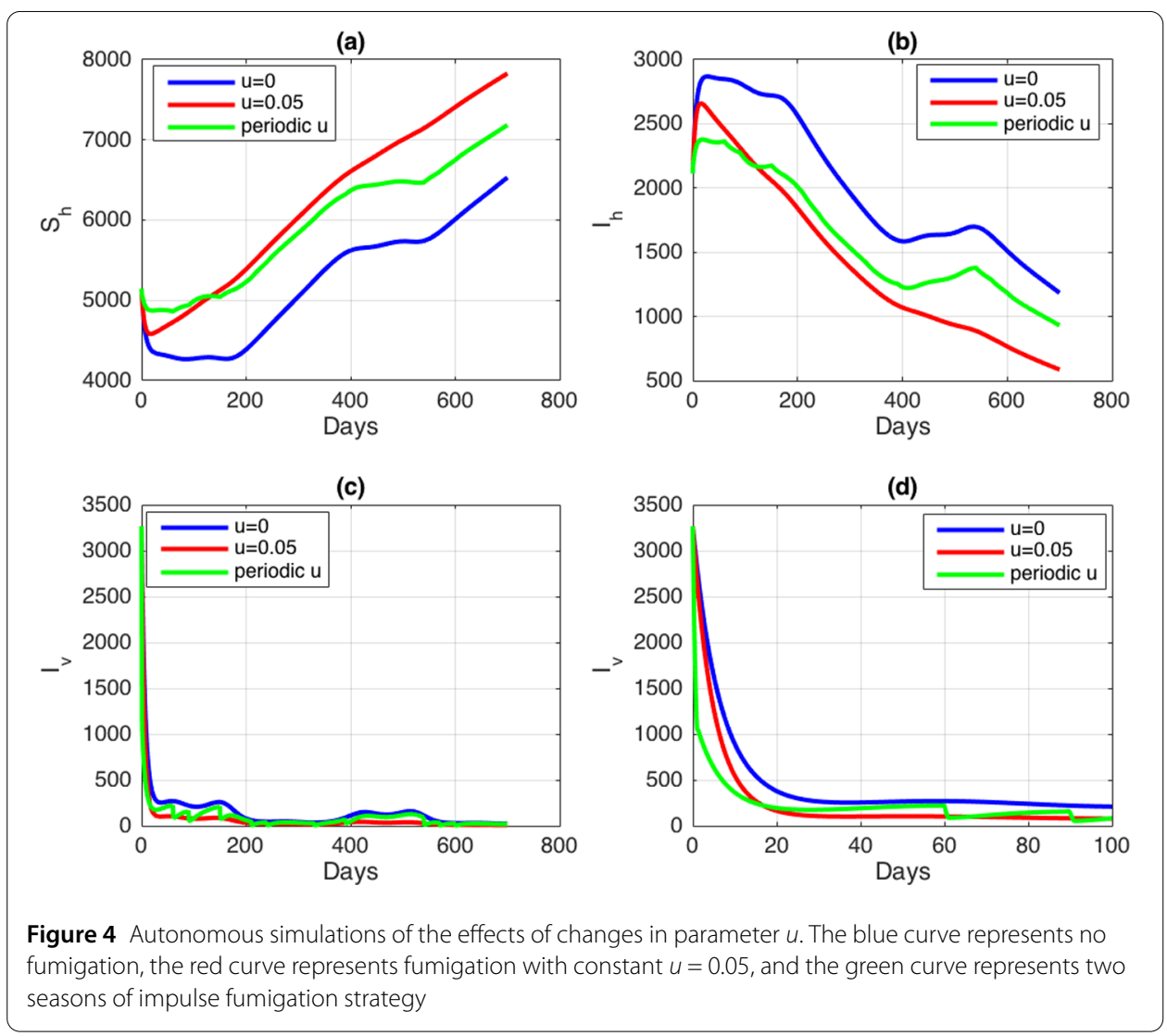




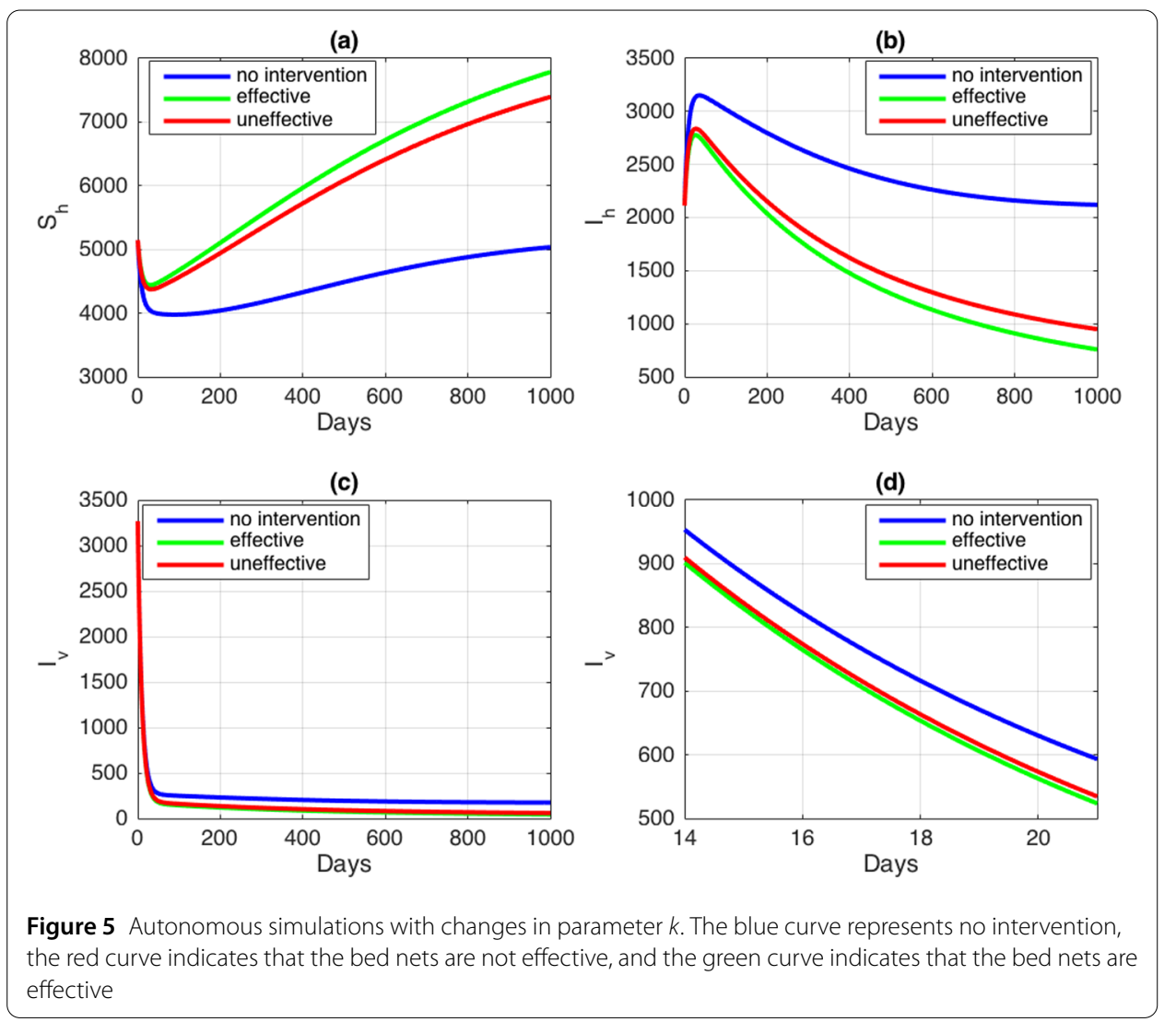

The use of bed nets in endemic areas is expected to be able to prevent the spread of malaria through mosquito bites. In this simulation, to suppress the number of infected people, we choose a situation where no fumigation is performed $(u=0)$. The autonomous simulation of bed nets is shown in Fig. 5 .

In Fig. 5, when the bed nets are used, the blue curve shows that malaria persists with $\mathcal{R}_{0}=1.412014007>1$. The red curve describes a population with bed nets but without taking into account the minimum quality $\eta$ of the bed nets, resulting in ineffectiveness with $\mathcal{R}_{0}=1.059010505>1$. However, if the value of $\eta$ is chosen based on the previous $\mathcal{R}_{0}$ sensitivity $(\eta=0.4)$, then, as seen in the green curve, the population is freed from malaria with $\mathcal{R}_{0}=0.9884098046<1$. Although the difference in $\mathcal{R}_{0}$ is not significant, overall, we can state that not only should the proportion $k$ of bed nets usage be considered, but also the quality $\eta$ of the bed nets should be considered. Note that bed nets are given to prevent mosquitos from biting humans, not to reduce the number of mosquitos. Therefore the difference in each scenario in the mosquito population is not significant.

\section{Malaria model with environment stochasticity}

In the previous sections, we used a deterministic model of malaria spread where all parameters are constant. In real-world conditions, there are environmental factors that are crucial to the spread of malaria, such as body temperature, $\mathrm{CO}_{2}$ levels released by humans, and residing near stagnant water; these factors are all changeable and unpredictable. Therefore, to observe the effect of random environmental factors on malaria spread, in this section, we consider the approach of stochastic differential equations (SDEs). 
Since the above environmental factors are related to the influence of the mosquito biting rate $\beta_{i}(k, \eta)$, a stochastic noise is introduced by replacing the parameter $\beta_{i}(k, \eta), i=h, v$, with

$$
\begin{aligned}
& \hat{\beta}_{h}(k, \eta) d t=\beta_{h}(k, \eta) d t+\sigma d W_{h}(t), \\
& \hat{\beta}_{v}(k, \eta) d t=\beta_{v}(k, \eta) d t+\sigma d W_{v}(t),
\end{aligned}
$$

where $\sigma$ is the noise intensity (a positive constant), and $d W_{i}(t)=W_{i}(t+d t)-W_{i}(t)$ is the increment of a Brownian motion (Higham [13]).

By substituting Eqs. (15) into the deterministic model (1) we obtain the following SDEs:

$$
\begin{aligned}
& d S_{h}=\left(\mu_{h} N_{h}-c_{h} \beta_{h}(k, \eta) \frac{S_{h}}{N_{h}} I_{v}-\mu_{h} S_{h}+\delta R_{h}\right) d t-\left(\sigma c_{h} \frac{S_{h}}{N_{h}} I_{\nu}\right) d W_{h}(t), \\
& d I_{h}=\left(c_{h} \beta_{h}(k, \eta) \frac{S_{h}}{N_{h}} I_{v}-\gamma I_{h}-\mu_{h} I_{h}\right) d t+\left(\sigma c_{h} \frac{S_{h}}{N_{h}} I_{v}\right) d W_{h}(t), \\
& d R_{h}=\left(\gamma I_{h}-\mu_{h} R_{h}-\delta R_{h}\right) d t \\
& d S_{v}=\left(\mu_{v} N_{v}-\left(c_{v} \beta_{v}(k, \eta) S_{v} \frac{I_{h}}{N_{h}}\right)-\mu_{v} S_{v}-u S_{v}\right) d t-\left(\sigma c_{v} S_{v} \frac{I_{h}}{N_{h}}\right) d W_{v}(t), \\
& d I_{v}=\left(c_{v} \beta_{v}(k, \eta) S_{v} \frac{I_{h}}{N_{h}}-\mu_{v} I_{v}-u I_{v}\right) d t+\left(\sigma c_{v} S_{v} \frac{I_{h}}{N_{h}}\right) d W_{v}(t) .
\end{aligned}
$$

Next, we performed numerical simulations to determine the effects of stochastic factors and the implications of parameter changes on the SDE model (16). Simulations were performed for as many as 250 iterations using the Euler-Maruyama method for 4096 days with two scenarios, a simulation of fumigation intervention and a simulation of bed net intervention. In the following simulation results, the first three curves are for human populations, and the last two curves are for mosquito populations.

Simulations of fumigation are divided into two cases, cases without (Figs. 6-7) and with (Fig. 8) time-dependent mosquito death rates. Since the simulations focus on fumigation, we set the parameter of the bed net proportion to $k=0$. Based on Eq. (10), the fumigation rate $u=0.02$ is selected for $\mathcal{R}_{0}>1$, and $u=0.06$ for $\mathcal{R}_{0}<1$. Figure 6 shows the conditions for endemic disease $\left(\mathcal{R}_{0}>1\right)$. The stochastic trends are quite different from the deterministic trends. The greater the value of noise intensity $\sigma$, the more significant the fluctuations in the simulations, which means that when an epidemic occurs, the greater environmental factors significantly affect the dynamics of all subpopulations. The results in Fig. 7 are quite different from those in Fig. 6, where the stochastic trend is approaching a deterministic trend. The noise intensity value does not significantly affect the fluctuations. In other words, when $\mathcal{R}_{0}<1$, environmental factors will not significantly affect the dynamics of the subpopulations. These situations appear because the environment stochasticity is included in the infection term $\left(\beta_{i}\right)$ in our model. When $\mathcal{R}_{0}>1$, infected compartments tend toward nonzero equilibrium, which makes it easy to capture the impact of $\sigma$. On the other hand, when $\mathcal{R}_{0}<1$, where all infected compartments tend toward the zero-equilibrium point, the impact of $\sigma$ is lower, and all variables significantly fluctuate.

For the second case, we consider the influence of the season on the mosquito death rate $\mu_{\nu}$ since the life expectancy of mosquitos during rainy and dry season is variable. A constant mosquitos death rate $\mu_{\nu}$ is converted to a function $\mu_{\nu}(t)$ in Eq. (14). The intervention 

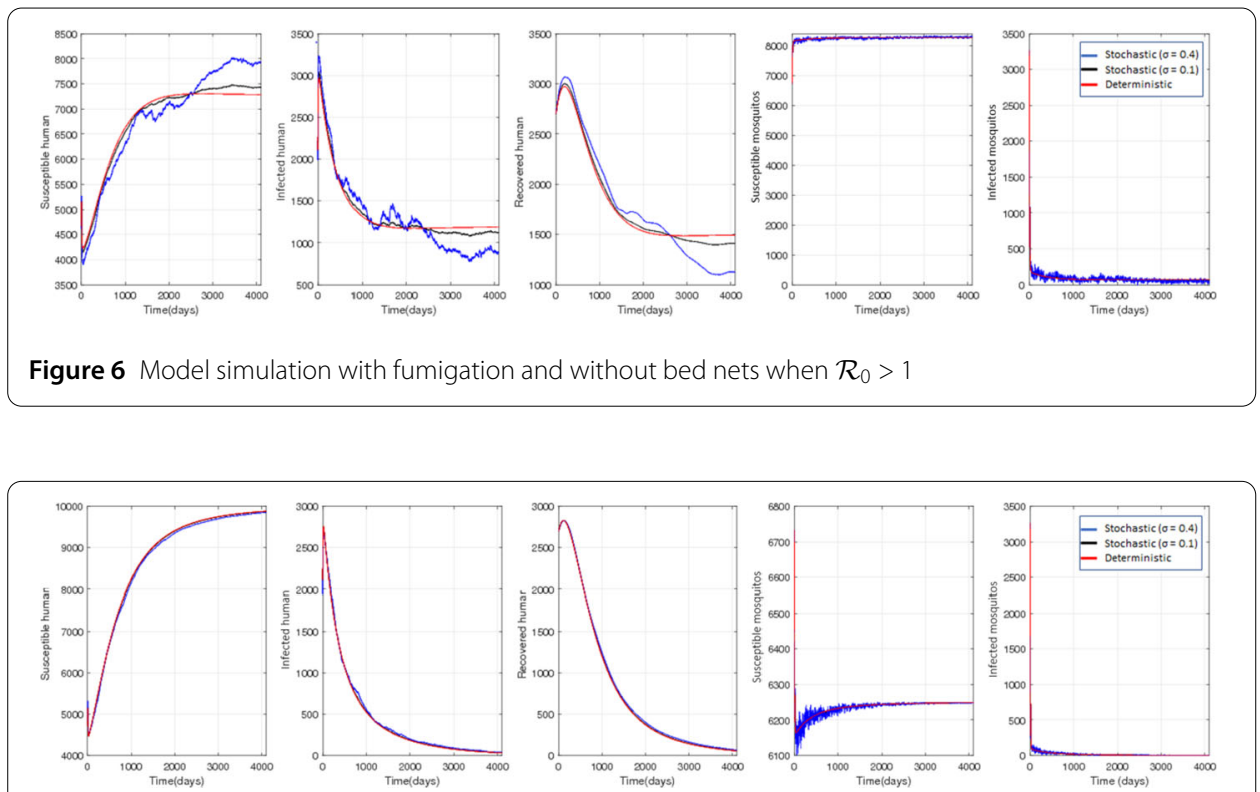

Figure 7 Model simulation with fumigation and without bed nets when $\mathcal{R}_{0}<1$
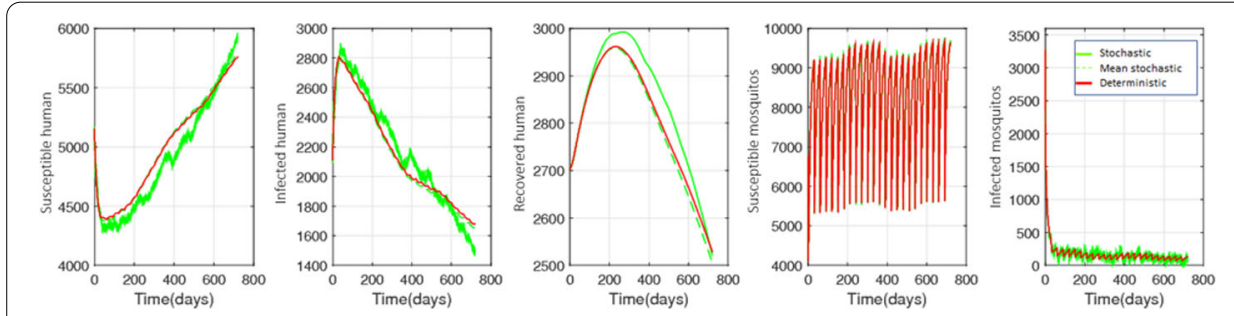

Figure 8 Model simulation with periodic fumigations and without bed nets with the influence of the season

is given on the first day of the month periodically for 720 days. In Fig. 8, the curves from susceptible mosquitos and infected mosquitos oscillate every 30 days due to the season and periodic fumigation. The dynamics of the human population also cause oscillations with smaller fluctuations than those of the mosquito population because the dynamics of the mosquito population dynamics gain stability more quickly than those of the human population. We refer to this situation as a fast dynamics and slow dynamics for mosquitos and humans, respectively, which means that when an epidemic of malaria occurs, the more random the environment, the more unpredictable the dynamics of malaria over a shortterm period.

Analyzing the effects of bed net intervention, we set the fumigation parameter $u$ equal to 0 . Based on Eq. (12), the proportion of bed nets is $k=0.5$, and the effectiveness of bed nets is $\eta=0.5$ for $\mathcal{R}_{0}>1$, whereas $\eta=0.4$ for $\mathcal{R}_{0}<1$. The results in Figs. 9 and 10 are not significant. In Fig. 10 the infected human and mosquito populations decrease slightly faster than in the corresponding population in Fig. 9 and tends toward zero because of the effectiveness of bed nets. This result shows that higher quality bed nets more effectively reduce the spread of malaria. This result is consistent with the results of the deterministic model in Fig. 5. 

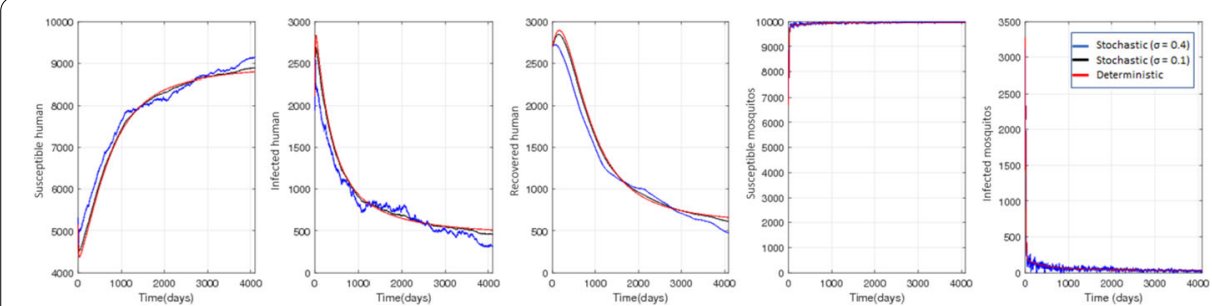

Figure 9 Model simulation with bed nets $\eta=0.5$ and without fumigation when $\mathcal{R}_{0}>1$
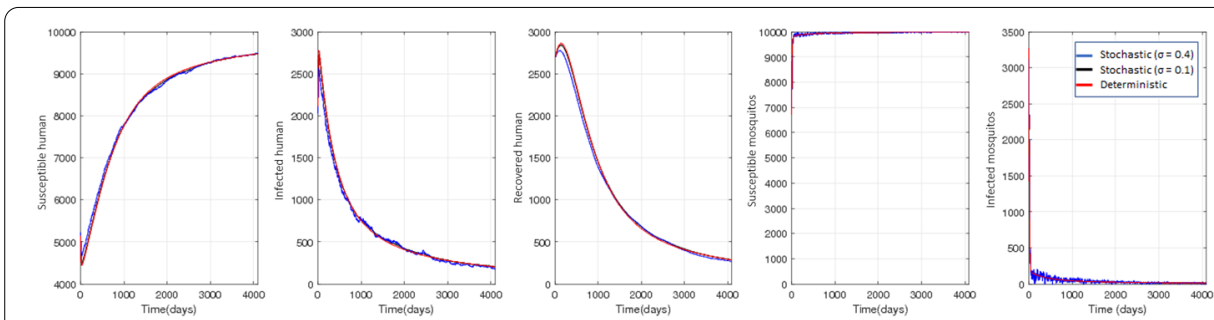

Figure 10 Model simulation with bed nets $\eta=0.4$ and without fumigation when $\mathcal{R}_{0}<1$
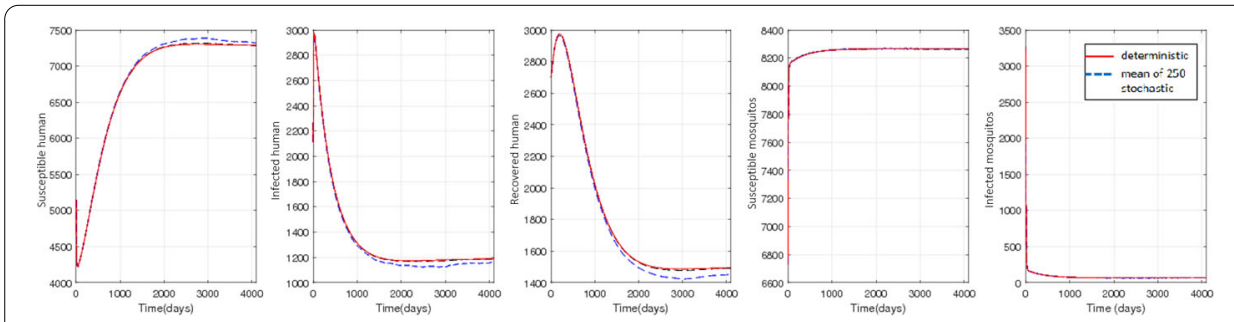

Figure 11 Model simulation with fumigation and without bed nets when $R_{0}>1$

Note that all above simulations result from one of the stochastic simulations. Of interest is that the mean of the 250 stochastic simulations shows a different performance. For example, in the simulation in Fig. 11 the mean solution is quite similar to the deterministic solution. In other words, the mean curve of the stochastic simulations is close to that of the deterministic model. The same results also have been mentioned in previous studies (Feng et al. [11]; Banks, Catenacci and $\mathrm{Hu}$ [5]). Simulations using other scenarios also have the same characteristics as simulations in Fig. 11.

\section{Optimal control problem}

There are several obstacles in using fumigation. One of these is the high cost of fumigation. To overcome this problem, the deterministic model (1) can be developed into an optimal control problem where the fumigation parameter $u$, which was previously set as a constant, now changes into a control variable $u(t)$ that depends on time. The purpose of this optimal control problem is determining the continuous piecewise function of the control variable $u(t)$ in the interval $t_{0}=0$ through $t_{1}=T$, which reduces infected populations at a minimum cost.

We assume the costs to reduce the numbers of infected individuals with malaria $I_{h}(t)$ and $I_{\nu}(t)$ are linear functions because of the assumption that the incurred costs are directly 
proportional to the numbers of infected humans and mosquitos. However, the cost of fumigation is a nonlinear function because a wider area of intervention causes a sharp, nonlinear cost increase. Therefore the objective function of the spread of malaria with fumigation can be written as follows:

$$
J(u(t))=\int_{0}^{T}\left(\sum_{i=1}^{5} \omega_{i} x_{i}(t)+\omega_{u} u(t)^{2}\right) d t
$$

where $x_{i}, i=1,2, \ldots, 5$, present $S_{h}, I_{h}, R_{h}, S_{v}$, and $I_{v}$, respectively. Since we only want to minimize the number of infected individuals, we set $\omega_{2}$ and $\omega_{5}$ greater than 0 , whereas the other parameters are set to 0 . Since higher numbers of fumigations elevate the intervention cost, we set $\omega_{v}>0$. Therefore we have

$$
J(u(t))=\int_{0}^{T}\left(\omega_{2} I_{h}(t)+\omega_{5} I_{v}(t)+\omega_{u} u(t)^{2}\right) d t
$$

We assume that the control $u(t)$ is not carried out all the time; instead, it is periodically used every $h$ days for $T$ days. Therefore $u(t)$ can be represented as a semidiscrete function (Wijaya et al. [21])

$$
\hat{u}_{\mathrm{opt}}(t)=\sum_{0 \leq j \leq\left\lfloor\left(\frac{T}{h}\right)\right\rfloor} \hat{u}(t) \mathbf{1}_{\left[t_{h, j}, t_{h, j}+1\right)}
$$

In Eq. (18), $\mathbf{1}_{\left[t_{h, j}, t_{h, j}+1\right)}$ is the characteristic function in the interval $\left[t_{h . j}, t_{h . j}+1\right)$, and $\hat{u}(t)$ is the value of the control variable at time $t$ given in Eq. (21).

Next, we define the Hamiltonian (Lenhart et al. [17]). The Hamiltonian consists of the sum of the integrand of the objective function in Eq. (17) and the inner products of the state in Eq. (1) with the adjoint variable $\lambda_{k}, k=1,2,3,4,5$. The Hamiltonian $\mathrm{H}\left(x_{i}, u, \lambda_{k}\right)$ can be stated as follows:

$$
\begin{aligned}
\mathrm{H}\left(x_{i}, u, \lambda_{k}\right)= & \left(\omega_{2} I_{h}(t)+\omega_{5} I_{v}(t)+\omega_{u} u(t)^{2}\right) \\
& +\lambda_{1}\left(\mu_{h} N_{h}-\frac{C_{h} \beta_{1}(k) S_{h}(t) I_{v}(t)}{N_{h}}-\mu_{h} S_{h}(t)+\delta R_{h}(t)\right) \\
& +\lambda_{2}\left(\frac{C_{h} \beta_{1}(k) S_{h}(t) I_{v}(t)}{N_{h}}-\gamma_{h} I_{h}(t)-\mu_{h} I_{h}(t)\right) \\
& +\lambda_{3}\left(-\delta R_{h}(t)+I_{h}(t) \gamma_{h}-R_{h}(t) \mu_{h}\right) \\
& +\lambda_{4}\left(\mu_{v} N_{v}-\frac{C_{v} \beta_{2}(k) I_{h}(t) S_{v}(t)}{N_{h}}-\mu_{v} S_{v}(t)-u(t) S_{v}(t)\right) \\
& +\lambda_{5}\left(\frac{C_{v} \beta_{2}(k) I_{h}(t) S_{v}(t)}{N_{h}}-\mu_{v} I_{v}(t)-u(t) I_{v}(t)\right) .
\end{aligned}
$$

Theorem 1 Given an optimal control variable $u(t)$ that minimizes objective function $J$ (17) and the solutions for $S_{h}, I_{h}, R_{h}, S_{v}, I_{v}$ of the corresponding system (1), there exist adjoint 
variables

$$
\begin{aligned}
& \dot{\lambda}_{1}=-\frac{\partial \mathrm{H}}{\partial S_{h}(t)}=\left(\lambda_{1}-\lambda_{2}\right)\left(\frac{C_{h} \beta_{1}(k) I_{v}(t)}{N_{h}}\right)+\lambda_{1} \mu_{h}, \\
& \dot{\lambda}_{2}=-\frac{\partial \mathrm{H}}{\partial I_{h}(t)}=\lambda_{2}\left(\gamma_{h}+\mu_{h}\right)-\lambda_{3} \gamma_{h}+\left(\lambda_{4}-\lambda_{5}\right)\left(\frac{C_{v} \beta_{2}(k) S_{v}(t)}{N_{h}}\right)-\omega_{2}, \\
& \dot{\lambda}_{3}=-\frac{\partial \mathrm{H}}{\partial R_{h}(t)}=\lambda_{3}\left(\delta+\mu_{h}\right)-\lambda_{1} \delta, \\
& \dot{\lambda}_{4}=-\frac{\partial \mathrm{H}}{\partial S_{v}(t)}=\left(\lambda_{4}-\lambda_{5}\right)\left(\frac{C_{v} \beta_{2}(k) I_{h}(t)}{N_{h}}\right)+\lambda_{4}\left(\mu_{v}+u(t)\right), \\
& \dot{\lambda}_{5}=-\frac{\partial \mathrm{H}}{\partial I_{v}(t)}=\left(\lambda_{1}-\lambda_{2}\right)\left(\frac{C_{h} \beta_{1}(k) S_{h}(t)}{N_{h}}\right)+\lambda_{5}\left(\mu_{v}+u(t)\right)-\omega_{5},
\end{aligned}
$$

with the transversality condition for adjoint variables

$$
\lambda_{k}(t)=0, \quad k=1,2,3,4,5 .
$$

Since the admissible control should be bounded with $u_{\min }$ and $u_{\max }$ as the lower and upper bounds of $u(t)$, respectively, the control variable $u(t)$ is represented by

$$
\hat{u}(t)=\min \left(u_{\max }, \max \left(u_{\min }, \frac{\lambda_{4} S_{v}(t)+\lambda_{5} I_{v}(t)}{2 \omega_{u}(t)}\right)\right) .
$$

Proof We first differentiate the negative of the Hamiltonian (19) with respect to each state variable, so the adjoint variables have the following form:

$$
\begin{aligned}
& \dot{\lambda}_{1}=-\frac{\partial \mathrm{H}}{\partial S_{h}(t)}=\left(\lambda_{1}-\lambda_{2}\right)\left(\frac{C_{h} \beta_{1}(k) I_{v}(t)}{N_{h}}\right)+\lambda_{1} \mu_{h}, \\
& \dot{\lambda}_{2}=-\frac{\partial \mathrm{H}}{\partial I_{h}(t)}=\lambda_{2}\left(\gamma_{h}+\mu_{h}\right)-\lambda_{3} \gamma_{h}+\left(\lambda_{4}-\lambda_{5}\right)\left(\frac{C_{v} \beta_{2}(k) S_{v}(t)}{N_{h}}\right)-\omega_{2}, \\
& \dot{\lambda}_{3}=-\frac{\partial \mathrm{H}}{\partial R_{h}(t)}=\lambda_{3}\left(\delta+\mu_{h}\right)-\lambda_{1} \delta, \\
& \dot{\lambda}_{4}=-\frac{\partial \mathrm{H}}{\partial S_{v}(t)}=\left(\lambda_{4}-\lambda_{5}\right)\left(\frac{C_{v} \beta_{2}(k) I_{h}(t)}{N_{h}}\right)+\lambda_{4}\left(\mu_{v}+u(t)\right), \\
& \dot{\lambda}_{5}=-\frac{\partial \mathrm{H}}{\partial I_{v}(t)}=\left(\lambda_{1}-\lambda_{2}\right)\left(\frac{C_{h} \beta_{1}(k) S_{h}(t)}{N_{h}}\right)+\lambda_{5}\left(\mu_{v}+u(t)\right)-\omega_{5},
\end{aligned}
$$

with the transversality condition for adjoint variables

$$
\lambda_{k}(T)=0, \quad k=1,2,3,4,5
$$

To obtain optimal conditions, we also differentiate the Hamiltonian (19) with respect to $u(t)$ and set these equations equal to zero:

$$
\frac{\partial \mathrm{H}}{\partial u(t)}=2 \omega_{u}(u(t))-\lambda_{4} S_{v}(t)-\lambda_{5} I_{v}(t)=0 .
$$


Solving (22) with respect to the control variable, we obtain

$$
u^{*}(t)=\frac{\lambda_{4} S_{v}(t)+\lambda_{5} I_{v}(t)}{2 \omega_{u}} .
$$

The control variable $u^{*}(t)$ in (23) must satisfy $u_{\min } \leq u^{*}(t) \leq u_{\max }$ for all $t \in[0, T]$, and then the control variable $\hat{u}(t)$ can be written as

$$
\hat{u}(t)=\min \left(u_{\max }, \max \left(u_{\min }, \frac{\lambda_{4} S_{v}(t)+\lambda_{5} I_{v}(t)}{2 \omega_{u}(t)}\right)\right),
$$

where $u_{\max }$ and $u_{\min }$ are the lower and upper bounds for the control variable, respectively.

Several scenarios are implemented in numerical simulations based on the results of Theorem 1 . The simulations are conducted using four different scenarios, that is, different values of $\mathcal{R}_{0}$ (e.g., seasonal influence, implementation of bed nets, and different initial conditions). To find a balance between each component in the cost function (17), we choose $\omega_{2}=0.5, \omega_{5}=0.0025$, and $\omega_{u}=0.01$. Note that our control variable is a rate of intervention, which can tend to $\infty$. Therefore we can choose the interval value of fumigation is $0 \leq u(t) \leq 5$ with the time interval of $0 \leq t \leq 500$ days. Fumigations are conducted periodically every 30 days for 500 days. It is assumed that one intervention will have an impact for the next three days. In numerical simulations we used the iterative gradient descent algorithm to accelerate the convergence of the control variable $u(t)$.

Numerical simulations are conducted with and without fumigation when $\mathcal{R}_{0}>1$ and $\mathcal{R}_{0}<1$ and for the purpose of comparing the reductions of infected humans and mosquitos. The $\mathcal{R}_{0}$ formula refers to Eq. (6). The initial values of each population are $S_{h}(0)=8000, I_{h}(0)=1900, R_{h}(0)=100, S_{v}(0)=8000$, and $I_{v}(0)=2000$.

Based on the results in Fig. 2, the biting rate value of mosquitos must satisfy $\beta_{h}<$ 0.17554563 to satisfy $\mathcal{R}_{0}<1$; thus we choose the parameter values $\beta_{h}=\beta_{v}=0.17$, which gives $\mathcal{R}_{0}=0.6868$. On the other hand, in the case where $\mathcal{R}_{0}>1$, which describes an epidemic that still exists in the environment, we have chosen the value for $\beta_{h}=\beta_{v}=0.35$, which gives the result for $\mathcal{R}_{0}=1.412$.

Figure 12 shows changes in the number of infected humans and mosquitos for 500 days. The dynamics of the control variables $u(t)$ in the cases of $\mathcal{R}_{0}>1$ and $\mathcal{R}_{0}<1$ have similar behavior; that is, when infected mosquitos exist, fumigation must be performed. However, when the number of infected mosquitos begins to decrease, the intervention must be reduced. The intervention in the case of $\mathcal{R}_{0}<1$ decreases faster due to rapidly reduced numbers of infected mosquitos compared to the case of $\mathcal{R}_{0}>1$. As a result, the cost function of $\mathcal{R}_{0}>1$ is $3.4632225 \times 10^{5}$, whereas in the case of $\mathcal{R}_{0}<1$, it is only $2.535228 \times 10^{5}$. This result appears because, under the condition $\mathcal{R}_{0}<1$, the environment most likely tends to a malaria-free equilibrium even though fumigation is not implemented. However, this fumigation policy faster achieves the malaria-free equilibrium. Therefore, the cost function when $\mathcal{R}_{0}<1$ is much lower than when $\mathcal{R}_{0}>1$.

In Table 3 , in the cases of $\mathcal{R}_{0}>1$ and $\mathcal{R}_{0}<1$, fumigation is equally successful in reducing the number of infected mosquito populations. In the case of $\mathcal{R}_{0}>1$ the reduction in the infected mosquito population on the 500th day after the intervention is $79.48 \%$, whereas 


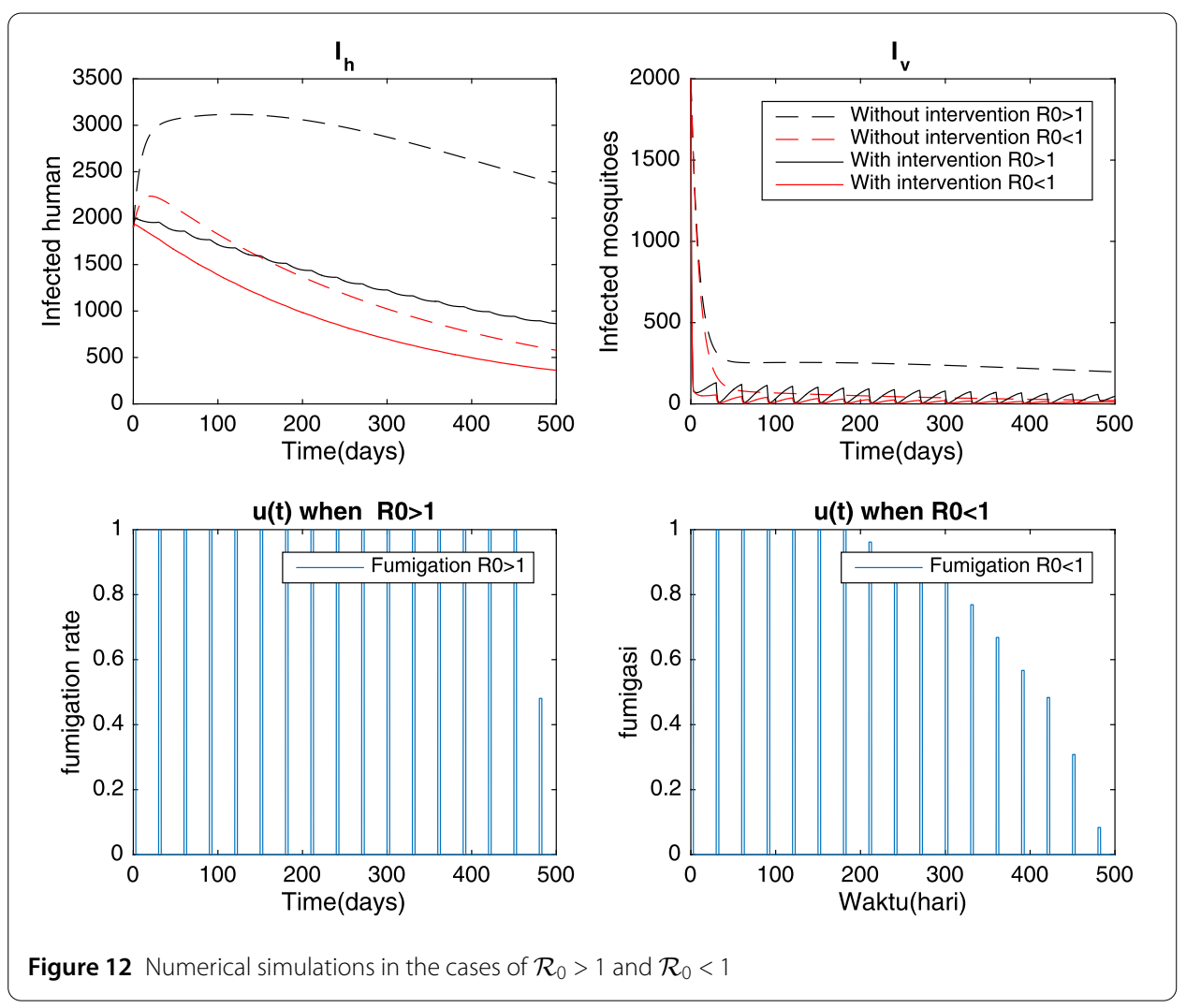

Table 3 Number of infected individuals on day 500 in the cases of $\mathcal{R}_{0}<1$ and $\mathcal{R}_{0}>1$

\begin{tabular}{llllllll}
\hline Population & $\mathcal{R}_{0}>1$ & & & & $\mathcal{R}_{0}<1$ \\
& Without & With & Percentage & & Without & With & \\
& intervention & intervention & reduction & intervention & intervention & $\begin{array}{l}\text { Percentage } \\
\text { reduction }\end{array}$ \\
\hline Infected mosquitos & 234 & 48 & & $\downarrow 79.48 \%$ & 21 & 13 & \\
Infected humans & 2367 & 865 & $\downarrow 63.45 \%$ & 577 & 362 & $\downarrow 38.05 \%$ \\
\hline
\end{tabular}

in the case of $\mathcal{R}_{0}<1$, it was $38.05 \%$. The results are similar for the number of infected humans.

In the following simulations, we aim to determine the dynamics for infected humans and mosquitos with and without fumigation by seasonal and nonseasonal influence. As in Sect. 2 , the mosquito mortality rate $\mu_{v}$, which was constant, is converted into a function as in Eq. (14).

Based on Figs. 13 and 14, a fumigation intervention is successful in reducing the number of infected humans and mosquitos, and the number of reductions is shown in Table 4. However, when the seasonal influence is considered, the intervention effects decrease significantly when the rainy season period ends $(t=182)$. The cost function when there is a seasonal influence is $3.3577 \times 10^{5}$, whereas when there is no seasonal influence, it is $3.4632225 \times 10^{5}$.

Next, we performed numerical simulations by combining fumigation with nonseasonal influences and the use of bed nets. The purpose is to see whether the use of bed nets can help to reduce the number of infected humans and mosquitos more effectively than fumigation alone. In this simulation, two cases of bed net use were considered; the first 

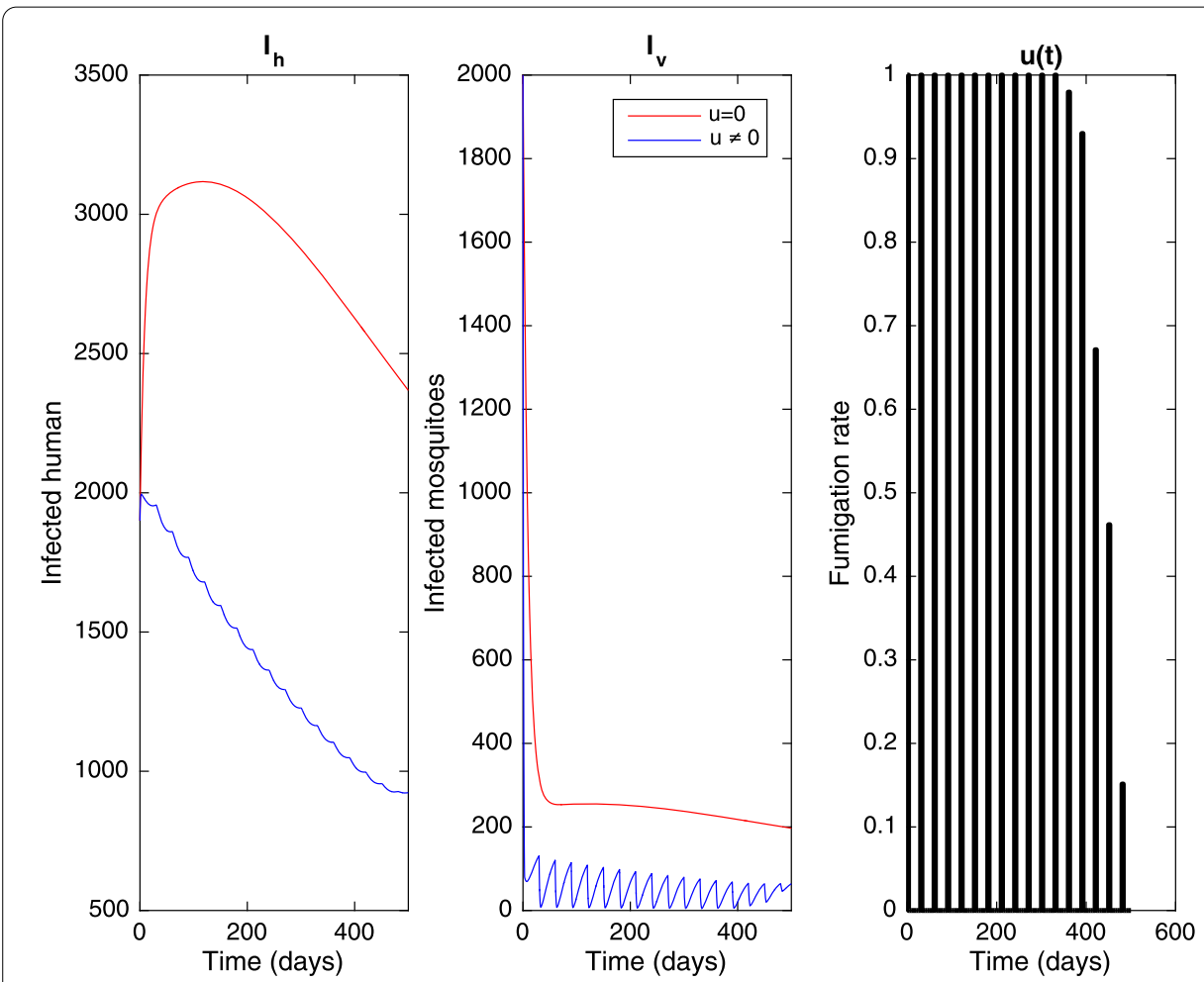

Figure 13 Numerical simulation results with and without fumigation in cases of nonseasonal influence
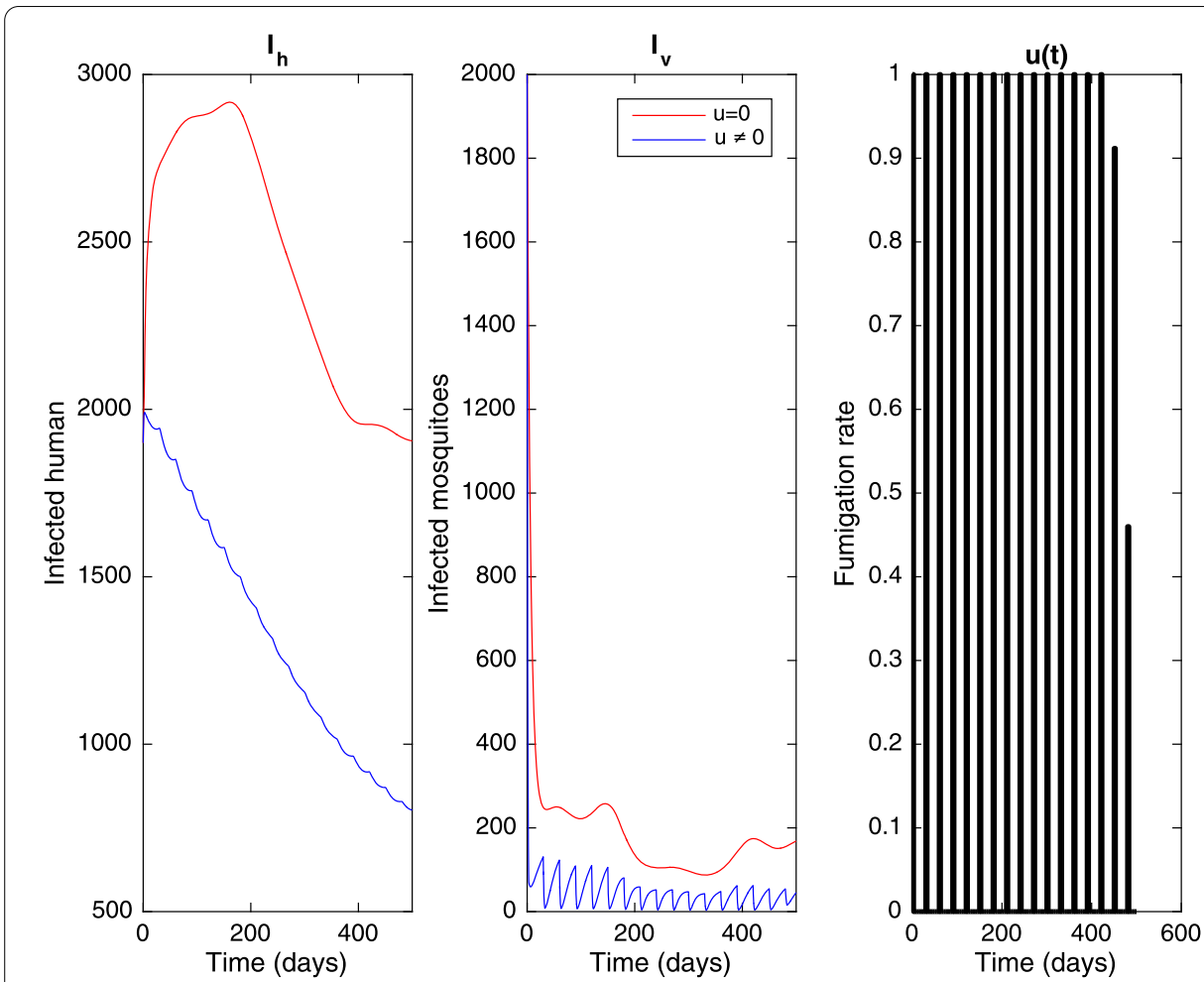

Figure 14 Numerical simulation results with and without fumigation in cases of seasonal influence 
Table 4 Number of humans and mosquitos infected on day 500 with seasonal influence

\begin{tabular}{|c|c|c|c|c|c|c|}
\hline \multirow[t]{2}{*}{ Populations } & \multicolumn{3}{|l|}{$\mathcal{R}_{0}>1$} & \multicolumn{3}{|l|}{$\mathcal{R}_{0}<1$} \\
\hline & $\begin{array}{l}\text { Without } \\
\text { intervention }\end{array}$ & $\begin{array}{l}\text { With } \\
\text { intervention }\end{array}$ & $\begin{array}{l}\text { Percentage } \\
\text { reduction }\end{array}$ & $\begin{array}{l}\text { Without } \\
\text { intervention }\end{array}$ & $\begin{array}{l}\text { With } \\
\text { intervention }\end{array}$ & $\begin{array}{l}\text { Percentage } \\
\text { reduction }\end{array}$ \\
\hline Infected mosquitos & 168 & 45 & $\downarrow 73.21 \%$ & 197 & 64 & $\downarrow 67.51 \%$ \\
\hline Infected humans & 1905 & 803 & $\downarrow 57.84 \%$ & 2367 & 923 & $\downarrow 61.00 \%$ \\
\hline
\end{tabular}
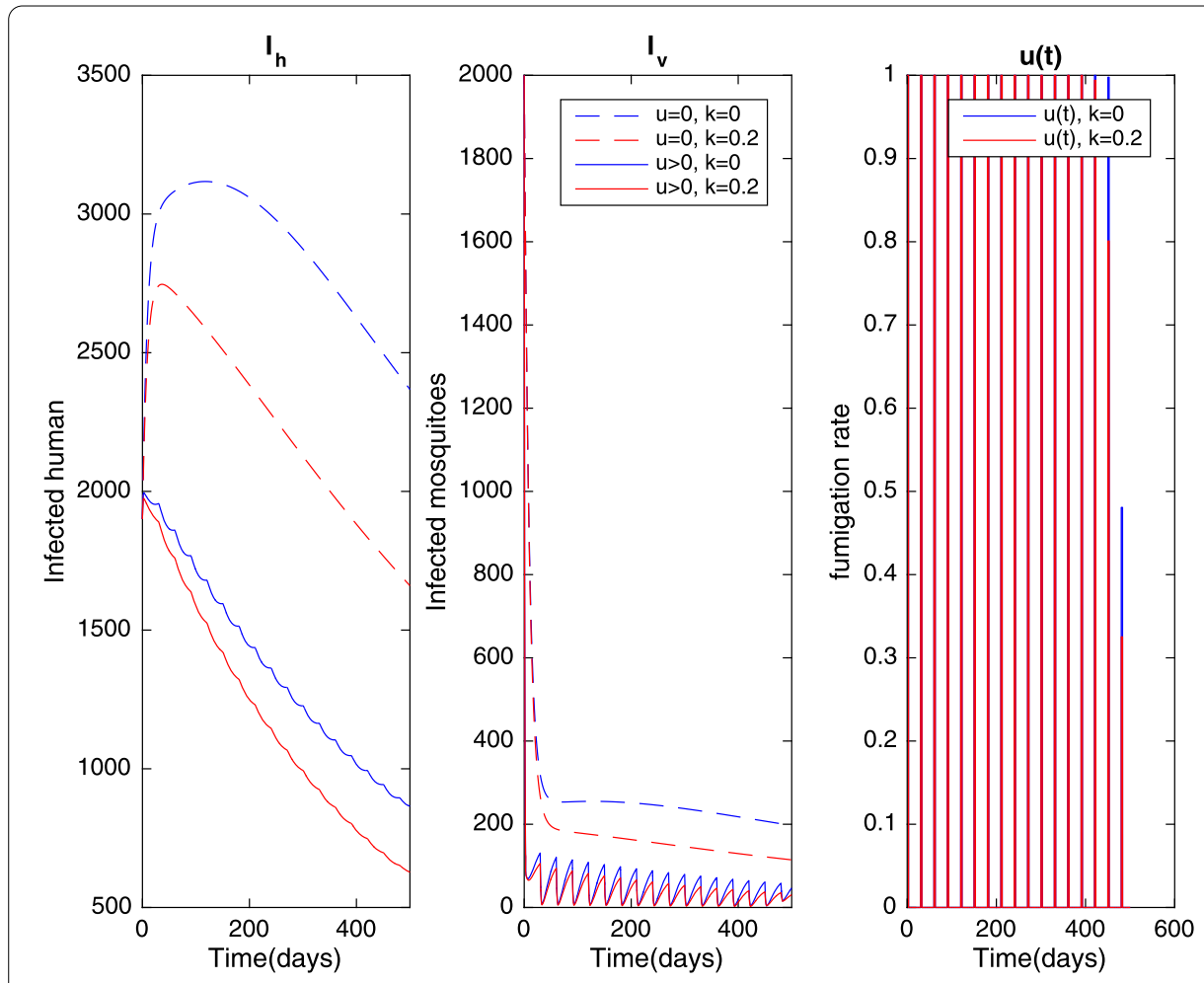

Figure 15 Results of simulations with and without fumigation intervention when $k=0$ and $k=0.2$

case is where there are no people using bed nets $(k=0)$, and in the second case, $20 \%$ of the total population uses bed nets $(k=0.2)$.

Changes in the number of infected humans and mosquitos and the dynamics of the control variable $u(t)$ can be seen in Fig. 15. The dynamics of the control variables show similar behavior in both cases. However, the fumigation used for case $k=0$ is greater than the intervention in the case of $k=0.2$. The cost function for the case of $k=0$ is $3.46322 \times 10^{5}$ compared to that for the case of $k=0.2$, which is $3.73323 \times 10^{5}$. Therefore the combination of bed nets and fumigation can reduce the number of infected mosquitos and humans with minimal cost compared to the numbers from the case without the combination.

Table 5 shows that the intervention of bed nets along with fumigation successfully reduces the number of infected humans and mosquitos. Even though using fumigation only for preventing malaria yielded a higher reduction of infected humans and mosquitos than using fumigation and bed nets together, the total number of infected individuals in case of fumigation and bed nets being used together is fewer than that achieved with fumigation only. This is highly related to how the use of bed nets can reduce $\mathcal{R}_{0}$. 
Table 5 The number of infected individuals on day 500 when $k=0$ and $k=0.2$

\begin{tabular}{|c|c|c|c|c|c|c|}
\hline \multirow[t]{2}{*}{ Population } & \multicolumn{3}{|l|}{$k=0$} & \multicolumn{3}{|l|}{$k=0.2$} \\
\hline & $\begin{array}{l}\text { Without } \\
\text { intervention }\end{array}$ & $\begin{array}{l}\text { With } \\
\text { intervention }\end{array}$ & $\begin{array}{l}\text { Percentage } \\
\text { reduction }\end{array}$ & $\begin{array}{l}\text { Without } \\
\text { intervention }\end{array}$ & $\begin{array}{l}\text { With } \\
\text { intervention }\end{array}$ & $\begin{array}{l}\text { Percentage } \\
\text { reduction }\end{array}$ \\
\hline Infected mosquitos & 168 & 45 & $\downarrow 73.21 \%$ & 114 & 31 & $\downarrow 72.8 \%$ \\
\hline Infected humans & 1905 & 803 & $\downarrow 57.84 \%$ & 1659 & 627 & $\downarrow 62.2 \%$ \\
\hline
\end{tabular}
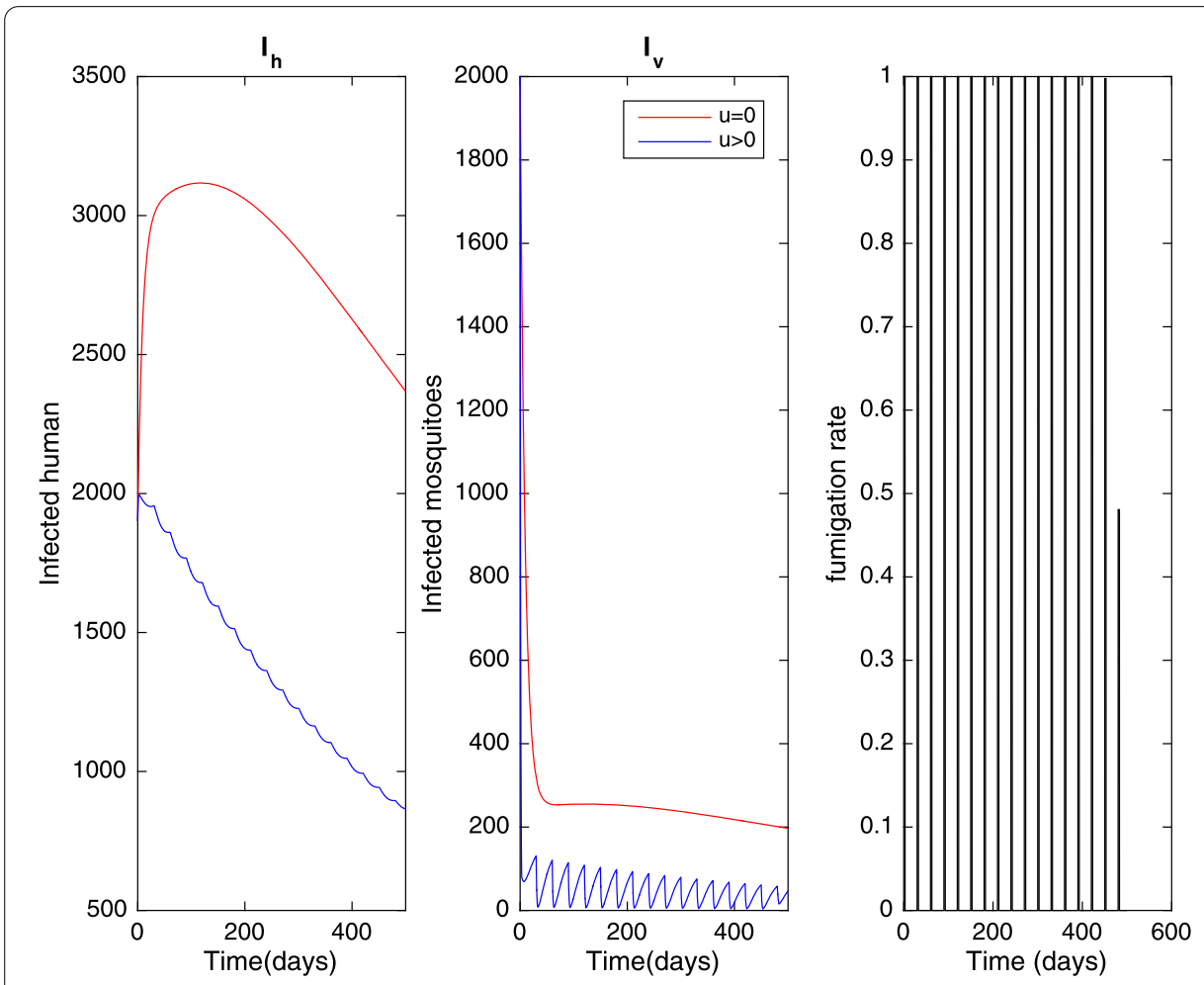

Figure 16 Numerical simulation results with and without fumigation in the endemic reduction scenario

Finally, we want to understand the influence of fumigation under two conditions that exist in the human population, endemic reduction and endemic prevention. The difference between the two conditions is the number of mosquitos and humans infected at the initial condition $t=0$, where in the endemic reduction scenario the number of infected individuals is relatively high as compared to the endemic prevention condition. Here we use initial conditions to illustrate the possible situations that occur in the field. In the endemic reduction scenario the initial values are $S_{h}(0)=8000, I_{h}(0)=1900, R_{h}(0)=100, S_{v}(0)=8000$, and $I_{\nu}(0)=2000$. In the endemic prevention scenario, the initial values are $S_{h}(0)=9900$, $I_{h}(0)=80, R_{h}(0)=20, S_{v}(0)=9950$, and $I_{v}(0)=50$.

The endemic reduction scenario illustrates cases that occur in the field when malaria infection has spread. The number of infected individuals (humans and mosquitos) and the dynamics of the control variable $u(t)$ are shown in Fig. 16. Table 6 shows the reduction in the number of infected individuals for this scenario after a period of time.

In the endemic prevention scenario the number of infected individuals is still relatively low (80), which describes malaria infections when it begins to spread. Changes in the number of infected individuals and the dynamics of the control variable $u(t)$ are shown 
Table 6 The numbers of each population of infected individuals on the 500th day of the endemic reduction scenario

\begin{tabular}{lccl}
\hline Population & $\begin{array}{l}\text { Without } \\
\text { intervention }\end{array}$ & $\begin{array}{l}\text { With } \\
\text { intervention }\end{array}$ & $\begin{array}{l}\text { Percentage } \\
\text { reduction }\end{array}$ \\
\hline Infected mosquitos & 168 & 45 & $\downarrow 73.21 \%$ \\
Infected humans & 1905 & 803 & $\downarrow 57.84 \%$ \\
\hline
\end{tabular}
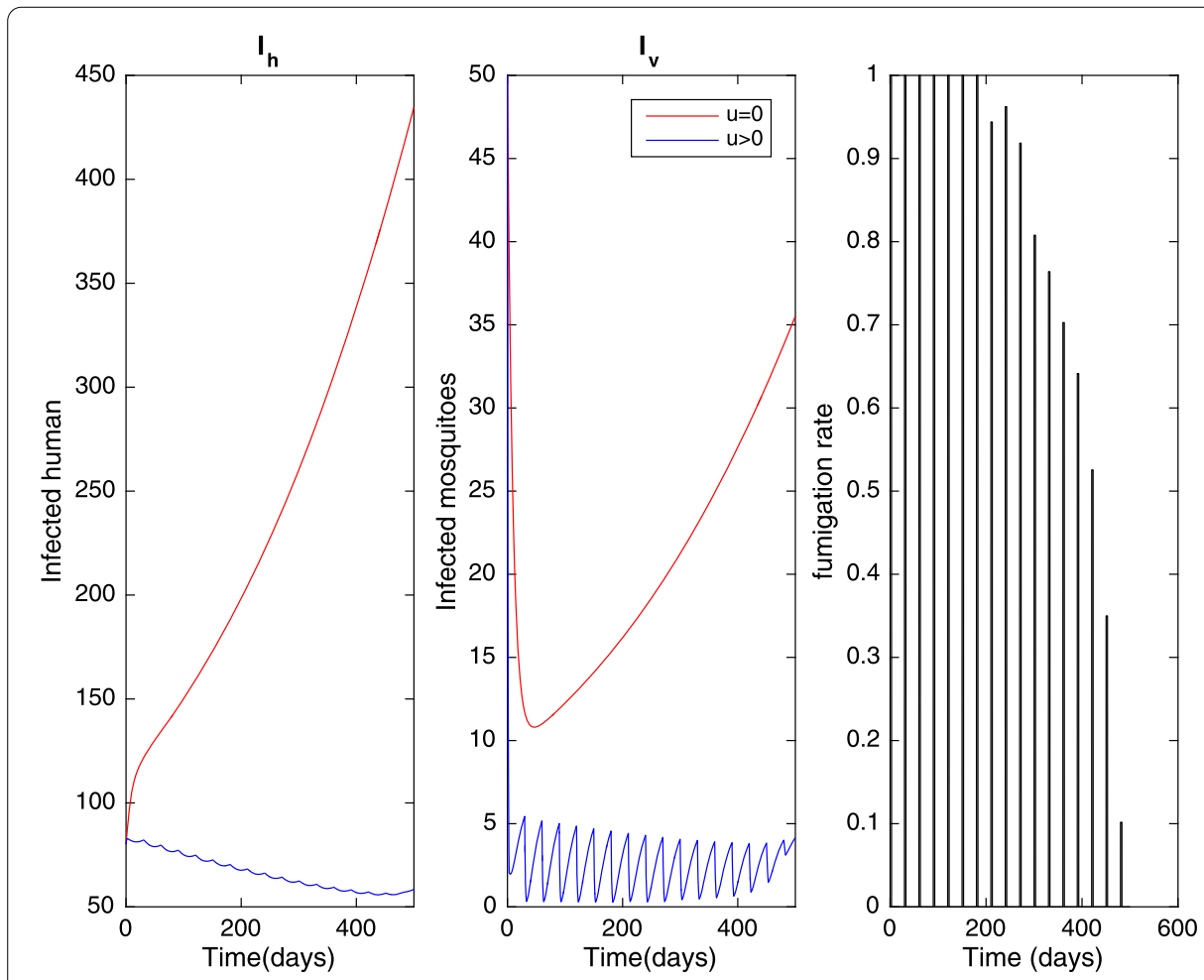

Figure 17 Numerical simulation results with and without fumigation in the endemic prevention scenario

Table 7 The number of each population of infected individuals on the 500th day of the endemic prevention scenario

\begin{tabular}{lccl}
\hline Population & $\begin{array}{l}\text { Without } \\
\text { intervention }\end{array}$ & $\begin{array}{l}\text { With } \\
\text { intervention }\end{array}$ & $\begin{array}{l}\text { Percentage } \\
\text { reduction }\end{array}$ \\
\hline Infected mosquitos & 35 & 5 & $\downarrow 85.71 \%$ \\
Infected humans & 434 & 59 & $\downarrow 86.41 \%$ \\
\hline
\end{tabular}

in Fig. 17. Table 7 contains information on the reduction numbers for infected individuals at the 500th day.

Based on Figs. 16 and 17, fumigation has successfully reduced the number of infected humans and mosquitos. The dynamic control variable $u(t)$ for both scenarios shows that for increase in the number of infected mosquitos, fumigation must be performed, and when the number of infected mosquitos begins to decrease, fumigation must be reduced. From the result of endemic prevention, the value of the intervention is $0<u(t)<2.5$, which is smaller than the results of the endemic reduction $0<u(t) \leq 5$. The cost function of the endemic reduction scenario is $3.46322 \times 10^{5}$, which is greater than the cost function for endemic prevention of $1.740746 \times 10^{4}$. This means that the prevention of malaria is much 
better if it is performed during the early stages of the endemic. Therefore an early warning system for malaria endemics should be considered to achieve better malaria prevention results.

Tables 6 and 7 show that the percentage reduction of the endemic prevention scenario is larger than that of the endemic reduction scenario. This is because the number of infected individuals to be reduced in an endemic reduction scenario is greater than that in an endemic prevention scenario.

\section{Conclusion}

The deterministic model of epidemic spread of malaria with bed nets and fumigation interventions has a malaria-free equilibrium (MFE), which always exists biologically, whereas the malaria endemic equilibrium (MEE) exists when $\mathcal{R}_{0}>1$. The MFE is asymptotically stable when $\mathcal{R}_{0}<1$, whereas MEE can be stated as a stable point under the condition $\mathcal{R}_{0} \geq 1$. The simulation results show that fumigation is not needed when the infection rate is less than the minimum boundary of the fumigation rate such that $\mathcal{R}_{0}<1$. In bed net interventions, when the proportion of bed nets used is less than the minimum value, bed nets will not eliminate malaria; thus the proportion of bed net users needs to be evaluated. Additionally, the better the quality of the bed nets, the higher the chance of reducing the number of mosquito bites. Simulations also show that when fumigation is not performed, many healthy humans become infected. Additionally, fumigation needs to be adjusted depending on season. By incorporating the seasonal influence the population of mosquitos reduces with $\mathcal{R}_{0}=0.9413426708<1$. However, with no intervention, $\mathcal{R}_{0}=1.412014006>1$; in other words, the malaria epidemic will still exist.

To observe the effect of random environmental factors on the spread of malaria, we considered a stochastic model. Numerical simulations were performed to determine the effects of stochastic factors and the implications of parameter changes on the model. The simulations show that when an epidemic exists, the environmental factors significantly affect the dynamics within all subpopulations. However, when $\mathcal{R}_{0}<1$, the environmental factors do not greatly affect the dynamics of the subpopulations.

When the intervention is performed every 30 days, the numbers of susceptible and infected mosquitos oscillate every 30 days due to the influence of the season and fumigation. Reducing the infected population requires using smaller values for the effectiveness of bed nets. These results are consistent with the analytic solution in the deterministic case.

To reduce the large cost of fumigation, the deterministic model was developed into an optimal control problem where fumigation parameters, which were previously set as constant, changed into control variables that depended on time. Fumigation was also conducted every 30 days, and we assumed that an intervention will have an impact until the following three days. The choice of parameter values also references the results of the deterministic case. The simulations show that when infected mosquitos exist, fumigation must be performed. However, when the number of infected mosquitos begins to decrease, the level of the intervention must be reduced. When seasonal influences are considered, the level of intervention decreases significantly when the rainy season ends. The cost function is lower with a seasonal influence than without it. Numerical simulations were also performed by combining fumigation and the use of bed nets, and the combination of both of these interventions can reduce the numbers of infected mosquitos and humans with minimal cost. The influence of fumigation in endemic reduction and endemic prevention 
was also investigated. Fumigation has successfully reduced the numbers of infected humans and mosquitos. The dynamic control variable shows that if the number of infected mosquitos increases, then fumigation is required; on the other hand, fumigation must be performed less frequently if there are fewer infected mosquitos. The cost function for the endemic reduction scenario is higher than the cost function for the endemic prevention scenario.

\section{Funding}

This research was financially supported by the Indonesia Ministry of Research and Higher Education (Kemenristek DIKTI) with PUPT research grant scheme 2018 with project ID No. 367/UN2.R3.1/HKP05.00/2018.

\section{Competing interests}

The authors declare that they have no competing interests.

Authors' contributions

All authors carried out the proofs of the main results and approved the final manuscript.

\section{Publisher's Note}

Springer Nature remains neutral with regard to jurisdictional claims in published maps and institutional affiliations.

Received: 10 December 2018 Accepted: 18 November 2019 Published online: 04 December 2019

\section{References}

1. Agusto, F.B., Valle, S.Y., Blayneh, K.W., et al.: The impact of bed-net use on malaria prevalence. J. Theor. Biol. 320, 58-65 (2013)

2. Aldila, D., Nuraini, N., Soewono, E.: Mathematical model in controlling dengue transmission with sterile mosquito strategies. AIP Conf. Proc. 1677, 030002 (2015)

3. Aldila, D., Padma, H., Khotimah, K., Handari, B.D., Tasman, H.: Analyzing the MERS control strategy through an optimal control problem. Int. J. Appl. Math. Comput. Sci. 28(1), 169-184 (2018)

4. Alemu, A., Abebe, G., Tsegaye, W., Golassa, L:: Climatic variables and malaria transmission dynamics in Jimma town, South West Ethiopia. Parasites Vectors 4(1), Article ID 30 (2011)

5. Banks, H.T., Catenacci, J., Hu, S.: A comparison of stochastic systems with different types of delays. Stoch. Anal. Appl. 31(6), 913-955 (2013)

6. Bustamam, A., Aldila, D., Yuwanda, A.: Understanding dengue control for short and long term intervention with a mathematical model approach. J. Appl. Math. 2018, Article ID 9674138 (2018)

7. CDC: Anopheles mosquitos. Centers for Disease Control and Prevention (2015)

8. Chitnis, N., Hardy, D., Gnaegi, G., et al.: Modeling the effects of vector control interventions in reducing malaria transmission, morbidity and mortality. Malar. J. 9(Suppl 2), Article ID 07 (2010)

9. Dembele, B., Friedman, A., Yakubu, A.: Malaria model with periodic mosquito birth and death rates. J. Biol. Dyn. 3(4), 430-445 (2009)

10. Diekmann, O., Heesterbeek, J.A.P.: Mathematical Epidemiology of Infectious Diseases: Model Building, Analysis and Interpretation. Wiley, New York (2000)

11. Feng, Z., Liu, R., Qiu, Z., Rivera, J., Yakubu, A.: Coexistence of competitors in deterministic and stochastic patchy environments. J. Biol. Dyn. 5(5), 454-473 (2011)

12. Gray, A., Greenhalgh, D., Hu, L., Mao, X., Pan, J.: A stochastic differential equation SIS epidemic model. SIAM J. Appl. Math. 71(3), 876-902 (2011)

13. Higham, D.J.: An algorithmic introduction to numerical simulation of stochastic differential equation. SIAM Rev. 43(3) 525-546 (2001)

14. Infodatin: Malaria. InfoDATIN (Pusat Data dan Informasi Kementerian Kesehatan RI). Kementerian Kesehatan RI, Jakarta (2016)

15. Keyser, H.: Why are some people more prone to mosquito bites? http://mentalfloss.com/article/57925/why-are-some-people-more-pronemosquito-bites (2014). Accessed 7 February 2018

16. Kim, S., Masud, M., Cho, G., Jung, I.H.: Analysis of a vector-bias effect in the spread of malaria between two different incidence areas. J. Theor. Biol. 419, 66-76 (2017)

17. Lenhart, S., Workman, J.T.: Optimal Control Applied to Biological Models. Chapman \& Hall/CRC Mathematical and Computational Biology Series. Chapman \& Hall/CRC, New York (2007)

18. Ngonghala, C.N., Del Valle, S.Y., Zhao, R., Mohammed-Awel, J., et al.: Quantifying the impact of decay in bed-net efficacy on malaria transmission. J. Theor. Biol. 363, 247-261 (2014)

19. Nkuo-Akenji, T., Ntonifor, N.N., Ndukum, M.B., Kimbi, H.K., Abongwa, E.L., Nkwescheu, A., Anong, D.N., Songmbe, M., Boyo, M.G., Ndamukong, K.N., Titanji, P.K., et al.: Environmental factors affecting malaria parasite prevalence in rural Bolifamba, South-West Cameroon. Afr. J. Health Sci. 13(1-2), 20-26 (2008)

20. Verhulst, F.: Nonlinear Differential Equation and Dynamical System, 2nd edn. Springer, Berlin (1990)

21. Wijaya, K.P., Goetz, T., Soewono, E., Nuraini, N.: Temephos spraying and thermal fogging efficacy on Aedes aegypti in homogeneous urban residences. ScienceAsia 39S, 48-56 (2013)

22. World Health Organization: Fact sheet about malaria. http://www.who.int/mediacentre/factsheets/fs094/en/ (2017). Accessed 1 February 2018 
23. World Health Organization: This year's world malaria report at a glance.

https://www.who.int/malaria/media/world-malaria-report-2018/en/ (2018). Accessed 21 April 2019

24. Wrede, R.C., Spiegel, M.R.: Schaums Outlines, Advanced Calculus. McGraw-Hill, New York (2010)

25. Xiunan, W., Xiao, Q.Z.: A Climate-Based Malaria Model with the Use of Bed Nets. Springer, Berlin (2017)

Submit your manuscript to a SpringerOpen ${ }^{\circ}$ journal and benefit from:

- Convenient online submission

- Rigorous peer review

- Open access: articles freely available online

- High visibility within the field

- Retaining the copyright to your article

Submit your next manuscript at $\gg$ springeropen.com 